OPEN ACCESS

Edited by:

Mora Murri,

University of Málaga, Spain

Reviewed by:

Maria Insenser,

Centre for Biomedical Network

Research (CIBER), Spain

Rosa Del Campo,

Ramón y Cajal Institute for Health

Research, Spain

*Correspondence:

Hsiang-Wen Lin

hsiangwl@gmail.com

Specialty section:

This article was submitted to

Translational Endocrinology,

a section of the journal

Frontiers in Endocrinology

Received: 18 June 2020

Accepted: 20 August 2020

Published: 23 September 2020

Citation:

Cao TTB, Wu K-C, Hsu J-L,

Chang C-S, Chou C, Lin C-Y,

Liao Y-M, Lin P-C, Yang L-Y and

Lin H-W (2020) Effects of Non-insulin

Anti-hyperglycemic Agents on Gut

Microbiota: A Systematic Review on

Human and Animal Studies.

Front. Endocrinol. 11:573891.

doi: 10.3389/fendo.2020.573891

\section{Effects of Non-insulin}

\section{Anti-hyperglycemic Agents on Gut Microbiota: A Systematic Review on Human and Animal Studies}

\author{
Thao T. B. Cao ${ }^{1,2}$, Kun-Chang Wu ${ }^{1}$, Jye-Lin $\mathrm{Hsu}^{3}$, Chih-Shiang Chang ${ }^{1}$, Chiahung Chou ${ }^{4,5}$, \\ Chen-Yuan Lin ${ }^{1,6}$, Yu-Min Liao ${ }^{6}$, Pei-Chun Lin ${ }^{1}$, Liang-Yo Yang ${ }^{7,8,9}$ and \\ Hsiang-Wen Lin ${ }^{1,10,11 *}$ \\ ${ }^{1}$ School of Pharmacy and Graduate Institute, China Medical University, Taichung City, Taiwan, ${ }^{2}$ Department of Clinical \\ Pharmacy, Hanoi University of Pharmacy, Hanoi, Vietnam, ${ }^{3}$ Graduate Institute of Biomedical Sciences, China Medical \\ University, Taichung City, Taiwan, ${ }^{4}$ Department of Health Outcomes Research and Policy, Harrison School of Pharmacy, \\ Auburn University, Auburn, AL, United States, ${ }^{5}$ Department of Medical Research, China Medical University Hospital, \\ Taichung City, Taiwan, ${ }^{6}$ Division of Hematology and Oncology, China Medical University Hospital, Taichung City, Taiwan, \\ ${ }^{7}$ Department of Physiology, School of Medicine, College of Medicine, China Medical University, Taichung City, Taiwan, \\ ${ }^{8}$ Laboratory for Neural Repair, China Medical University Hospital, Taichung City, Taiwan, ${ }^{9}$ Biomedical Technology Research \\ and Development Center, China Medical University Hospital, Taichung City, Taiwan, ${ }^{10}$ Department of Pharmacy, China \\ Medical University Hospital, Taichung City, Taiwan, " Department of Pharmacy System, Outcomes and Policy, College of \\ Pharmacy, University of Illinois at Chicago, Chicago, IL, United States
}

Background: As growing evidence links gut microbiota with the therapeutic efficacy and side effects of anti-hyperglycemic drugs, this article aims to provide a systematic review of the reciprocal interactions between anti-hyperglycemic drugs and gut microbiota taxa, which underlie the effect of the gut microbiome on diabetic control via bug-host interactions.

Method: We followed the PRISMA requirements to perform a systematic review on human vs. animal gut microbiota data in PubMed, SCOPUS, and EMBASE databases, and used Cochrane, ROBIN-I, and SYRCLE tools to assess potential bias risks. The outcomes of assessment were trends on gut microbiota taxa, diversity, and associations with metabolic control (e.g., glucose, lipid) following anti-hyperglycemic treatment.

Results: Of 2,804 citations, 64 studies (17/humans; 47/mice) were included. In human studies, seven were randomized trials using metformin or acarbose in obese, pre-diabetes, and type 2 diabetes (T2D) patients. Treatment of pre-diabetes and newly diagnosed T2D patients with metformin or acarbose was associated with decreases in genus of Bacteroides, accompanied by increases in both Bifidobacterium and Lactobacillus. Additionally, T2D patients receiving metformin showed increases in various taxa of the order Enterobacteriales and the species Akkermansia muciniphila. Of seven studies with significant differences in beta-diversity, the incremental specific taxa were associated with the improvement of glucose and lipid profiles. In mice, the effects of metformin on A. muciniphila were similar, but an inverse association with Bacteroides was reported. Animal studies on other anti-hyperglycemic drugs, however, showed substantial variations in results. 
Conclusions: The changes in specific taxa and $\beta$-diversity of gut microbiota were associated with metformin and acarbose in humans while pertinent information for other anti-hyperglycemic drugs could only be obtained in rodent studies. Further human studies on anti-hyperglycemic drugs other than metformin and acarbose are needed to explore gut microbiota's role in their therapeutic efficacies and side effects.

Keywords: anti-hyperglycemic drugs, microbiome, microbiota, association, systematic review

\section{INTRODUCTION}

Gut microbiota plays a pivotal role in the pathogenesis of diabetes as significant alterations were found in the gut microbiome composition in type 2 diabetes (T2D) patients relative to healthy individuals (1). A metagenome-wide association study reported a moderate degree of dysbiosis associated with depletion in butyrate-producing bacteria, accompanied by increases in opportunistic pathogens among diabetic patients (2). These changes were echoed by a recent systematic review, which shows an inverse association of T2D with the genera Bifidobacterium, Akkermansia and butyrate-producing bacteria (e.g., Roseburia, Faecalibacterium), in conjunction with a positive association with Ruminococcus, Fusobacterium, and Blautia (1).

From a clinical perspective, these findings provide a rationale for targeting gut microbiota imbalance as a potential strategy for T2D treatment by restoring a healthy gut microbiome, including fecal microbiota transplant and probiotic supplements $(3,4)$. However, the efficiency and effectiveness of these treatments remain uncertain due to concerns over the invasive nature of fecal microbiota transplant and the dosage, species, and duration required for an effective probiotic treatment. Emerging evidence indicates that the therapeutic efficacy of antihyperglycemic drugs might, in part, be attributable to their ability to modulate the compositions of gut microbiota $(1,3,5-9)$. This compositional change might lead to enrichments in bacterial species exhibiting beneficial effects to intestinal health via the production of health-promoting metabolites, such as short-chain fatty acids (SCFAs) and bile acids (8). Nevertheless, certain anti-hyperglycemic drugs were reported to cause increases in the abundance of Escherichia and Candidatus Arthromitus, which contribute to gastrointestinal side effects and weight gain, respectively (9-11).

Among various anti-hyperglycemic drugs in clinical use, metformin, acarbose, sitagliptin, and vildagliptin $(5,6)$ have been investigated for their reciprocal interplay with gut microbiota by assessing their effects on human and animal gut microbiota, and vice versa $(1,8,11)$. From a translational perspective, animal models might help to explore the causality of complex host-microbiota interactions and possible mechanisms of action in a controlled experimental setting. However, it should be noted that differences in dietary habits, host metabolism, inflammatory states, and body anatomy contribute to great variations in gut microbiota compositions between humans and animals, and subsequently, the respective drug effects in disease control (12). A meta-analysis of published 16S rDNA sequencing data from mouse and human fecal microbiota showed that there were significant increases in Lactobacillus and Turicibacter genera in mouse gut microbiota while the genera of Streptococcus, Ruminococcus, Lachnospira, Faecalibacterium, Dialister, and Oscillospira were elevated in human gut microbiota (12). Moreover, age, mouse strains/populations, microbiota pools in laboratories, and other practical factors might have varied to a great extent among different studies (12). Previous reviews have suggested the effects of anti-hyperglycemic drugs on gut microbiota $(8,11,13)$, however, the differences in results between human and animal studies have not been differentiated.

Reciprocal interplays between individual anti-hyperglycemic drugs and gut microbiota remain unexplored with respect to the contribution of specific bacterial taxa to drug's therapeutic efficacy in disease control (i.e., the clinical question). Thus, we conducted this systematic review aiming to shed light on the associations among anti-hyperglycemic agents, changes in specific taxonomic groups of gut microbiota, and host glucose control or metabolic profiles mainly in humans, as compared to those reported in animal studies.

\section{METHODS}

\section{Literature Search}

Our literature search strategies were designed to integrate the following PICOS (population, intervention, comparisons, outcomes, study design) based on the prior clinical question: Population: humans (e.g., healthy people or patients who were either obese, prediabetes, diabetes) or the corresponding animal models; Intervention: non-insulin anti-hyperglycemic drugs; Comparisons: post- vs. pre-intervention, with-vs. without-treatment, or on-vs. off-treatment; Outcomes: alteration of the gut microbial composition; Study design: clinical trials, observational studies or animal experiments, as that recommended for systematic reviews (14). We systematically searched PubMed, EMBASE, and SCOPUS databases from January 1, 2000, to November 13, 2019. The keywords and searching strategies based upon the PICOS were "antihyperglycemic drugs" and "gut microbiota" related terms (Supplementary Table 1). In addition, we searched manually the reference list of the review papers for additional publications of interest.

\section{Study Selection Criteria}

We followed the preferred reporting items for systematic reviews and meta-analysis (PRISMA) guidelines (15). The inclusion criteria for the published studies included: (i) any human studies or animal experiments reporting original data of gut microbiota 
after receiving anti-hyperglycemic drugs; (ii) gut microbiota data were analyzed from feces or colonic content specimens; (iii) must be written in English or Chinese. Studies were excluded if they did not provide data of individual bacterial taxa or were only available as conference abstracts or proceedings.

\section{Selection of Studies}

Initially, the abstracts and titles of potential articles were screened, followed by the evaluation of the full-text articles for eligibility. Two authors were responsible for screening and evaluating these papers independently. Disagreements were resolved by consensus between these two authors and, if necessary, discussed by additional two authors.

\section{Data Extraction}

A standardized form in a Microsoft Excel file (e.g., characteristics of studies, participants, treatments and comparisons, methods to analyze the microbiome, and measures of outcomes) was used for data extraction. Data were extracted by one author and reviewed by a second one. All disagreements were resolved by consensus and a third or fourth author when necessary.

\section{Quality Assessment}

We used the Cochrane risk-of-bias tool to assess the risk of bias in selected randomized trials (16). For quasi-experimental and observational studies, we used the Risk of Bias in Nonrandomized studies-of Interventions (ROBIN-I) to assess the risk of bias (17). Further, the SYRCLE's risk of bias tool for animal studies (18) was used to assess the risk of bias. The risks of bias data were extracted by four different authors and all disagreements were resolved by consensus made by the remaining authors.

\section{Outcomes of Assessment}

Other than describing the characteristics of the evaluated human or animal studies, the primary outcome was the difference in relative abundance or change patterns of individual intestinal bacterial taxa, categorized based on six common taxonomic categories [Phylum $(\mathrm{P}) \mathrm{c}$, Class $(\mathrm{C})$, Order $(\mathrm{O})$, Family $(\mathrm{F})$, Genus (G), Species (S)], in associations with the use of antihyperglycemic agents, among those available human or animal studies, respectively. Secondary outcomes were differences in microbial diversity, changes in intestinal or serum levels of SCFAs and/or bile acids in human or animal hosts, respectively, after taking/using individual drugs, associations between specific taxa and host metabolic parameters, e.g., glucose, body weight, and lipid profile.

\section{Data Synthesis}

We classified the primary and secondary outcomes into the following categories: significant increase, significant decrease, and no significant difference between comparison groups. Changes of each taxon were synthesized from at least 2 studies for human or animal studies, respectively. Specifically, the effects of different anti-hyperglycemic drugs on specific taxon among the evaluated human or animal studies were compared. Further, the corresponding effects of each individual drug on specific taxon were compared to explore its consistency, in terms of having the same trend of alteration on the specific taxon caused by the same specific anti-hyperglycemic drug, or not. These findings also were categorized by the target research populations (e.g., obese, prediabetic, newly T2D, prevalent T2D), individual treated drugs or different animal models (mice or rat models with various diets or genetic knockout). For gut microbial diversity, each study might use one or more measures to assess $\alpha$ - (richness and evenness) or $\beta$-diversity. We considered $\alpha$-diversity as "Increase" if at least one measure showed an increase and no measure showed a decrease; "Decrease" if at least one measure showed a decrease and no measure showed an increase; "No difference" if all measures showed no difference. $\beta$-diversity was assessed as "Difference" if at least one measure showed a difference; "No difference" if all measures showed no difference. In terms of associations between specific taxa and host metabolic parameters, we collected data from specific taxa that increased or decreased significantly in participants receiving non-insulin anti-hyperglycemic drugs. Only data with statistical significance were extracted for analyses.

\section{RESULTS}

The following presented results were mainly focused on human studies, which are compared to those of animal studies.

\section{Reviewed Studies}

Overall, 2,804 citations were retrieved, and the final analysis included 17 human studies $(7,19-34)$ and 47 mouse studies (5, 6, 35-79) from 64 papers (Figure 1). The majority of studies were published in and after 2017 and the duration of anti-hyperglycemic treatments varied from studies to studies (i.e., from few days to several months). Of 17 human studies, seven $(41.2 \%)$ were randomized control trials $(7,19-21,32-$ 34). Thirteen studies (76.4\%) enrolled either newly diagnosed or prevalent T2D patients $(20,21,23,24,26-34)$, whereas the remaining four studies enlisted healthy participants $(22,25)$, obese individuals (19), and pre-diabetic patients (7) (Tables 1, 2).

Of 47 rodent studies, $30(63.8 \%)$ studies were conducted in mice $(35-41,44-46,48,52,53,55,56,59-65,68,70,74-$ $79)$ and the others were in rats $(5,6,42,43,47,49-51$, $54,57,58,66,67,69,71-73)$. Their characteristics, housing, acclimatization, and diet treatments were presented in Table 3 and Supplementary Table 2. Overall, 14 anti-hyperglycemic agents were used in these included studies, with metformin accounting for the most. There was only one human study focused on glipizide, while the other ten listed drugs (e.g., voglibose, miglitol, vildagliptin, sitagliptin, saxagliptin) were used only in rodent studies (Table 1).

\section{Microbiome Assessment Method}

Fecal specimens of all human studies were analyzed for the composition of gut microbiota. Of 47 mouse studies, 34 studies (72.3\%) used fecal samples $(5,6,35,36,38,41,42,44-46,49-56$, 59-61, 63-73, 76, 78). 16S rRNA gene sequencing was the most common method used in human and animal studies (Table 1). 


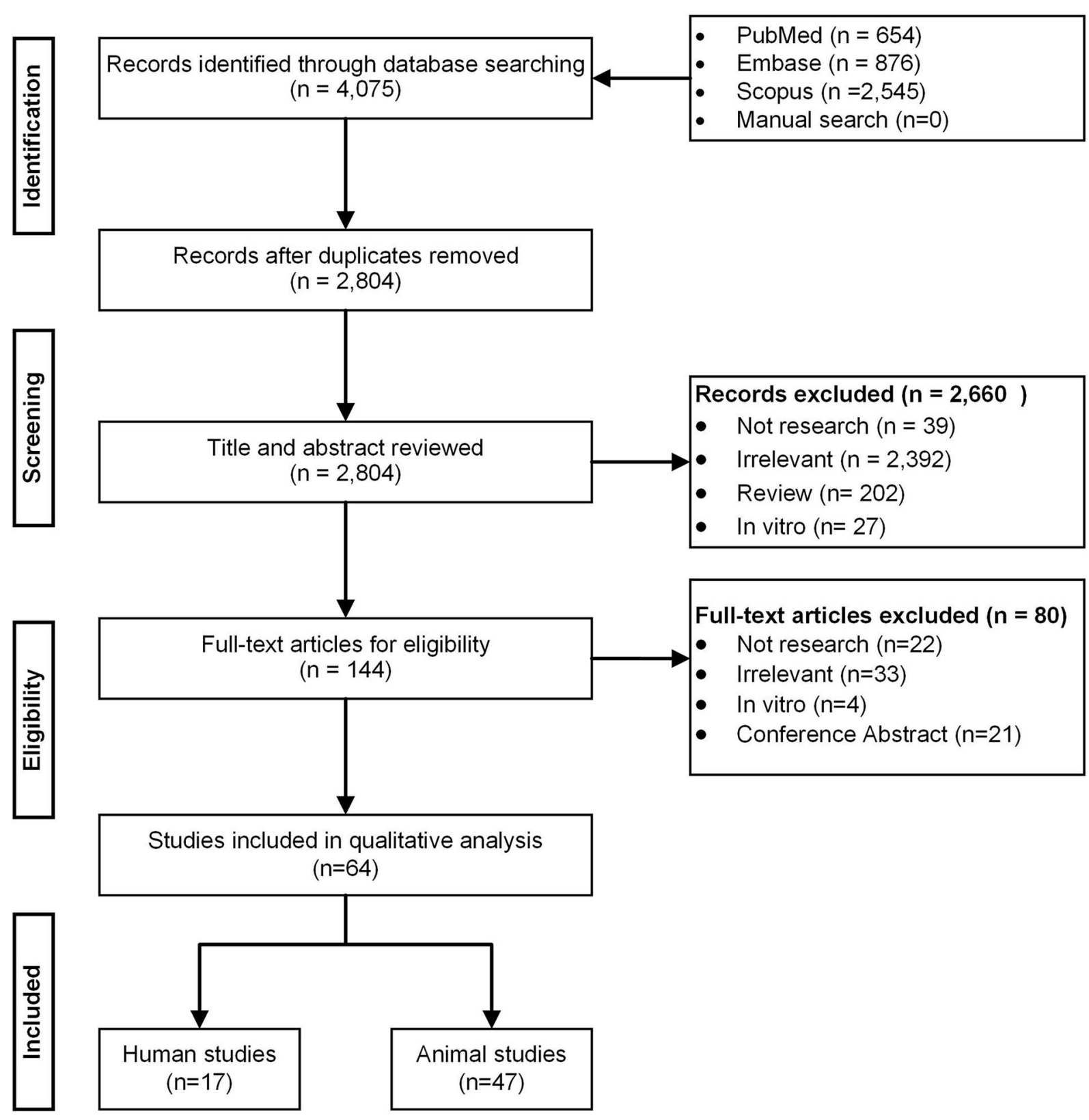

FIGURE 1 | PRISMA flow diagram.

\section{The Risk of Bias}

Among human studies, three randomized studies were at the high risk of bias in performance, detection, and attrition, while four studies were unclear risks in most domains (Supplementary Figure 1). Among quasi-experimental studies, three studies were at low risk of bias in all domains, whereas two studies were at serious risk in confounding, selection of participants, and classification of interventions (Supplementary Figure 2). All 5 cross-sectional studies were at serious risk in several domains, e.g., confounding, selection of participants, classification of intervention
(Supplementary Figure 3). Almost all mouse studies were unclear risk across domains, even if some were at low risk of bias in selective outcome reporting (Supplementary Figure 4).

\section{Outcomes of Assessment}

\section{Bacterial Taxa}

Importantly, the synthesized results from animal studies reported all common six taxonomic categories (P, C, O, F, G, S) of bacterial taxa but there were only three common taxonomic categories (F, $\mathrm{G}, \mathrm{S}$ ) of gut microbiota taxa were reported in those from human studies based on the available data (Tables 4-7). Glipizide and 
TABLE 1 | General characteristics of all included studies.

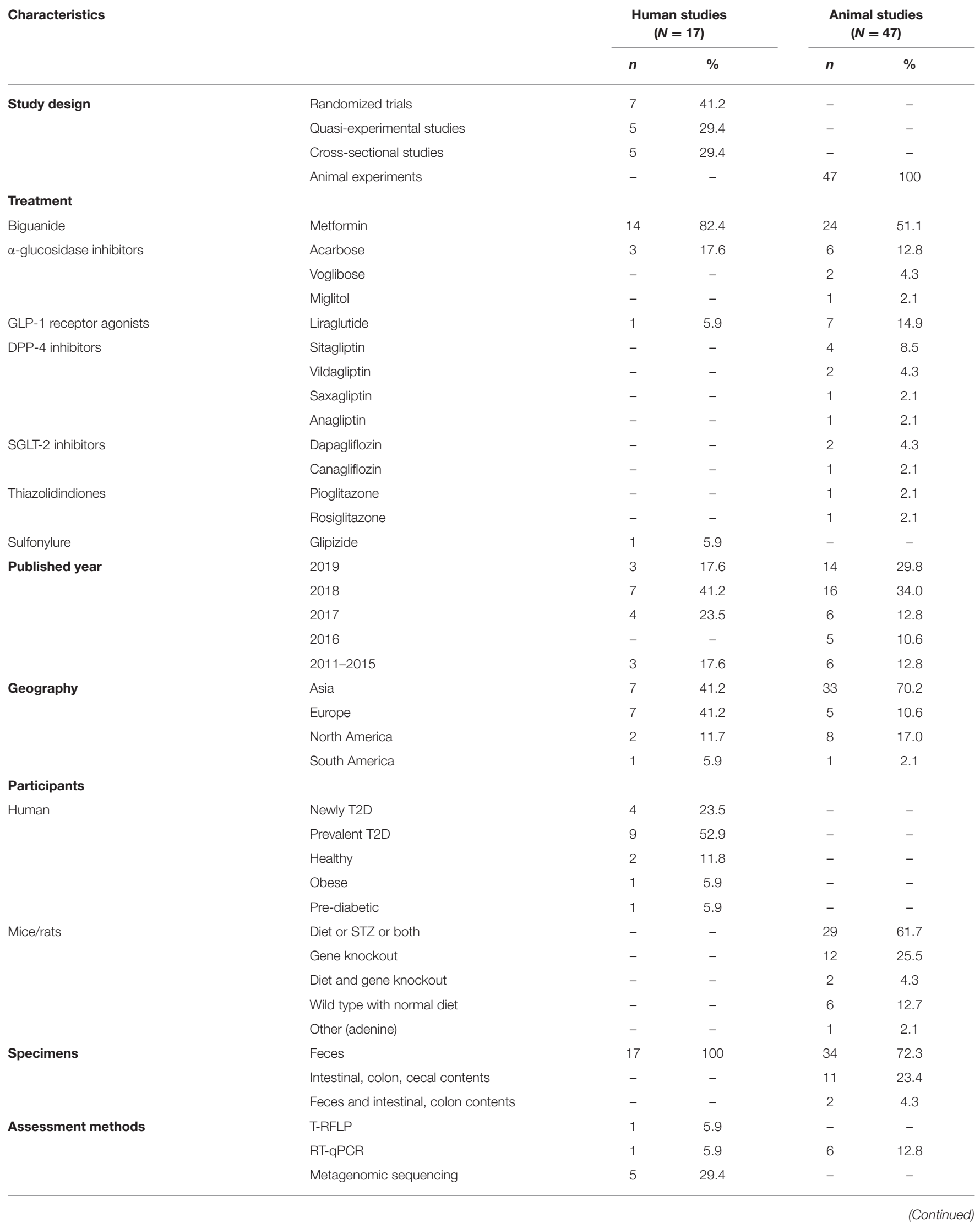


TABLE 1 | Continued

\begin{tabular}{|c|c|c|c|c|c|}
\hline \multirow[t]{2}{*}{ Characteristics } & & \multicolumn{2}{|c|}{$\begin{array}{l}\text { Human studies } \\
\qquad(N=17)\end{array}$} & \multicolumn{2}{|c|}{$\begin{array}{c}\text { Animal studies } \\
(N=47)\end{array}$} \\
\hline & & $n$ & $\%$ & $n$ & $\%$ \\
\hline & 16S rRNA gene sequencing & 10 & 58.8 & 35 & 74.4 \\
\hline & 16S rRNA gene sequencing and metagenomic & - & - & 1 & 2.1 \\
\hline & 16S rDNA gene sequencing & - & - & 3 & 6.4 \\
\hline & DGGE & - & - & 1 & 2.1 \\
\hline & Cultivation & - & - & 1 & 2.1 \\
\hline \multirow[t]{10}{*}{ Variable gene region for gene sequencing } & V3-V4 & 2 & 11.8 & 15 & 31.9 \\
\hline & V4 & 3 & 17.6 & 9 & 19.1 \\
\hline & V3 & 1 & 5.9 & 4 & 8.5 \\
\hline & $\mathrm{V} 1-\mathrm{V} 2$ & 1 & 5.9 & 3 & 6.4 \\
\hline & $V_{1}-V_{3}$ & - & - & 5 & 10.6 \\
\hline & V1, V2, V3 & 1 & 5.9 & - & - \\
\hline & V3-V5 & 1 & 5.9 & - & - \\
\hline & V4-V5 & - & - & 1 & 2.1 \\
\hline & V5-V6 & - & - & 1 & 2.1 \\
\hline & Not stated & 1 & 5.9 & 1 & 2.1 \\
\hline
\end{tabular}

T-RFLP, terminal restriction fragment length polymorphism; RT-qPCR, Real-time quantitative polymerase chain reaction; DGGE, denaturing gradient gel electrophoresis; -, no information.

liraglutide were assessed in a single human study. No differences were found in patients treated with glipizide (32), while Wang et al. (34) found the association between liraglutide treatment and the increased abundance of genus Akkermansia in T2D patients. The assessments of the effects of metformin and acarbose on the human gut microbiota composition represented the foci of 14 studies $(19-31,34)$ and three studies $(7,32,33)$, respectively (Tables 4, 5).

Of the phylum Bacteroidetes, the genus Bacteroides decreased in two studies treated with metformin among newly diagnosed T2D patients $(20,24)$, and in two studies treated with acarbose among pre-diabetic and newly diagnosed T2D patients $(7,32)$. Additionally, one study treated with metformin (24) and one study treated with acarbose (32) in newly diagnosed T2D patients reported similar results of decreases in seven species (e.g., Bacteroides dorei, Bacteroides finegoldii). For the phylum Firmicutes, the genus Lactobacillus increased in two studies in pre-diabetic (7) and newly diagnosed T2D patients (32) receiving acarbose, and the species L. gasseri increased in one study treated with metformin (21), and with acarbose (32), respectively, among newly diagnosed T2D patients. Meanwhile, the genus Clostridium decreased in one study among healthy participants receiving metformin (22) which was also reported in newly diagnosed T2D patients treated with acarbose (32) (Table 4). Two species, i.e., C. bartlettii and C. botulinum, consistently decreased among T2D patients receiving metformin in two separate studies $(21,31)$. Three out of four studies showed a decrease in the genus Intestinibacter (21, 22, 30) among healthy participants and T2D patients treated with metformin (Table 5). With respect to the phylum Actinobacteria, the genus Bifidobacterium with the species B. adolescentis increased in one study with metformin (21) and another study with acarbose (32) among newly diagnosed T2D patients (Table 4), and B. longum consistently increased among T2D patients treated with acarbose in two studies $(32,33)$ (Table 5).

Concerning other phyla, two studies evaluated the genus Fusobacterium (phylum Fusobacteria) $(20,27)$ and the species Akkermansia muciniphila (phylum Verrucomicrobia) $(21,29)$ among T2D patients treated with metformin. Both showed increases in the abundance of these two taxa. In the phylum Proteobacteria, conflicting results were reported in two studies with respect to the family Enterobacteriaceae in healthy participants vs. T2D patients treated with metformin (25, 27). Overall, metformin might increase different taxa from the family Enterobacteriaceae and other families in the order of Enterobacteriales. Of six studies that evaluated the genus Escherichia $(19-22,25,30)$ and four studies evaluating the genus Shigella $(19,20,22,25)$, the treatment of healthy participants, obese individuals, and T2D patients with metformin all led to increased abundance of these two genera. Another two studies on metformin in newly diagnosed and prevalent T2D patients $(21,31)$ reported consistently increases in eight species of the order Enterobacteriales, including Citrobacter koseri, Escherichia coli, Klebsiella pneumonia (family Enterobacteriaceae), Erwinia amylovora (family Erviniaceae), Pectobacterium wasabiae, and Dickeya dadantii (family Pectobacteriaceae) (Table 5).

The effects of 13 anti-hyperglycemic drugs on the compositions of gut microbiota were conducted in different rodent models. The results of pioglitazone was inconclusive (78), while the other 12 drugs were presented in Tables 6, 7. The Firmicutes/Bacteroidetes ratios were decreased in two studies treated with metformin in high fat diet (HDF)-fed mice $(37,48)$. 
TABLE 2 | Characteristics of included human studies.

\begin{tabular}{ccc}
\hline Taxonomic $\quad \begin{array}{c}\text { SCFAs } \quad \text { Bile acids } \\
\text { composition }\end{array}$ & $\beta$ - diversity \\
\hline
\end{tabular}

\section{Randomized trials $(n=7)$}

\begin{tabular}{|c|c|c|c|c|c|c|c|c|c|c|c|c|}
\hline Ejtahed et al. (19), Iran & Obese & $20 / 16$ & $\begin{array}{l}\text { M (1,000 mg) vs. } \\
\text { placebo }\end{array}$ & $2 m$ & Feces & $16 \mathrm{~S}$ rRNA & Post vs. pre & $\sqrt{ }$ & $\sqrt{ }$ & $\sqrt{ }$ & $\sqrt{ }$ & \\
\hline Tong et al. (20), China & Newly T2D & $100 / 100$ & $\begin{array}{l}\text { Chinese medicine vs. M } \\
\text { ( } 750 \mathrm{mg})\end{array}$ & $12 w$ & Feces & $\begin{array}{l}\text { 16S rRNA V3-V4 } \\
\text { region }\end{array}$ & Post vs. pre & $\sqrt{ }$ & $\sqrt{ }$ & $\sqrt{ }$ & & \\
\hline \multirow[t]{2}{*}{ Wu et al. (21), Europe } & Newly T2D & $22 / 18$ & $\begin{array}{l}\text { M (425-1,700 mg) vs. } \\
\text { placebo }\end{array}$ & $4 \mathrm{~m}$ & Feces & $\begin{array}{l}\text { DNA shotgun } \\
\text { metagenomics }\end{array}$ & Post vs. pre & & $\sqrt{ }$ & $\sqrt{ }$ & & $\sqrt{ }$ \\
\hline & & & & & & & With vs. without & & & & $\sqrt{ }$ & $\sqrt{ }$ \\
\hline Zhang et al. (7), China & Pre-diabetes & $40 / 40$ & $\begin{array}{l}\text { A }(50-150 \mathrm{mg}) \text { vs. } \\
\text { placebo }\end{array}$ & $4 w$ & Feces & $\begin{array}{l}\text { 16S rRNA V3-V5 } \\
\text { region }\end{array}$ & Post vs. pre & $\sqrt{ }$ & $\sqrt{ }$ & $\sqrt{ }$ & & \\
\hline Gu et al. (32), China & Newly T2D & $51 / 43$ & $\begin{array}{l}\text { A ( } 75-450 \mathrm{mg}) \text { vs. G } \\
(5-15 \mathrm{mg})\end{array}$ & $3 m$ & Feces & $\begin{array}{l}\text { DNA } \\
\text { metagenomics }\end{array}$ & Post vs. pre & $\sqrt{ }$ & & $\sqrt{ }$ & & $\sqrt{ }$ \\
\hline Su et al. (33), China & Prevalent T2D & $59 / 36$ & A 150 mg vs. non-A & $4 w$ & Feces & $\begin{array}{l}16 \mathrm{~S} \text { rDNA } \\
\text { RT-qPCR }\end{array}$ & Post vs. pre & & & $\sqrt{ }$ & & \\
\hline \multirow[t]{2}{*}{ Wang et al. (34), USA } & Prevalent T2D & $19 / 18$ & L vs. M (as usual) & $18 w$ & Feces & $\begin{array}{l}16 \mathrm{~S} \text { rRNA V4 } \\
\text { region }\end{array}$ & Lvs. M & $\sqrt{ }$ & $\sqrt{ }$ & $\sqrt{ }$ & & \\
\hline & & & & & & & Post vs. pre & & $\sqrt{ }$ & $\sqrt{ }$ & & \\
\hline
\end{tabular}

Quasi-experimental studies ( $n=5$ )

Bryrup et al. (22), Denmark Healthy

$25 \quad$ M (500-2,000 mg)

$6 w$

Feces

16S rRNA V4

Post vs. pre

region

Huang et al. (23), Sweden

\section{Prevalent T2D}

23/7 M (as usual) vs. non-M

$28 w$

Sun et al. (24), China

Newly T2D

22

M $(2,000 \mathrm{mg})$

Elbere et al. (25), Latvia

Healthy

$18 \quad M(1,700 \mathrm{mg})$

Napolitano et al. (26), UK

Prevalent T2D

14/14 on-M (as usual) vs. off-M

$3 d$

Feces

16S rRNA gene

T-RFLP

DNA

metagenomics

With vs. without

Post vs. pre

16S rRNA

Post vs. pre

region

NA Feces 16S rRNA V1, V2, On vs. off $\mathrm{V} 3$ regions

\section{Cross-sectional studies $(n=5)$}

Zhang et al. (27), China

Prevalent T2D

non-treatment

Barengolts et al. (28), USA Prevalent T2D 25/16 M (as usual) vs. non-M

De La Cuesta-Zuluaga et al. Prevalent T2D $\quad 14 / 14 \quad$ M (as usual) vs. non-M

(29), Columbia

Forslund et al. (30), Denmark Prevalent T2D 58/17 M (as usual) vs. non-M

Karlsson et al. (31), Sweden Prevalent T2D 20/33 M (as usual) vs. non-M

$\begin{array}{lll}- & \text { Feces } & \begin{array}{l}\text { 16S rRNA V1-V2 } \\ \text { region } \\ \text { 16S rRNA V3-V4 } \\ \text { region } \\ \text { 16S rRNA V4 } \\ \text { region }\end{array} \\ - & \text { Feces } & \begin{array}{l}\text { 16S rDNA } \\ \text { shotgun } \\ \text { metagenomics } \\ \text { DNA }\end{array} \\ & \text { Feces } \\ & \text { Feces } & \end{array}$

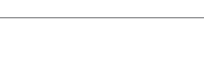

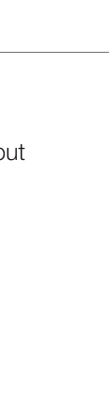

$\begin{array}{lll}\sqrt{ } & \sqrt{ } & \sqrt{ } \\ \sqrt{ } & & \sqrt{ } \\ \sqrt{ } & \sqrt{ } & \sqrt{ } \\ \sqrt{ } & \sqrt{ } & \sqrt{ } \\ & \sqrt{ } & \end{array}$

A, acarbose; G, glipizide; M, metformin; L, liraglutide; T-RFLP, terminal restriction fragment length polymorphism; RT-qPCR, Real-time quantitative polymerase chain reaction; $m$, months; $w$, weeks; $d$, days; -, no information; NA, not available; vs., versus. 
TABLE 3 | Characteristics of included animal studies.

\begin{tabular}{|c|c|c|c|c|c|c|c|c|c|c|c|c|c|}
\hline \multirow[t]{2}{*}{ References, country } & \multirow[t]{2}{*}{ Animals } & \multirow[t]{2}{*}{ Models } & \multirow[t]{2}{*}{$N$} & \multirow{2}{*}{$\begin{array}{l}\text { Treatment and } \\
\text { daily dose }\end{array}$} & \multirow[t]{2}{*}{ Duration } & \multirow[t]{2}{*}{ Specimens } & \multirow{2}{*}{$\begin{array}{l}\text { Analysis } \\
\text { methods }\end{array}$} & \multirow[t]{2}{*}{ Comparison } & \multicolumn{5}{|c|}{ Outcomes } \\
\hline & & & & & & & & & $\alpha$-diversity & $\beta$-diversity & $\begin{array}{l}\text { Taxonomic } \\
\text { composition }\end{array}$ & SCFAs & $\begin{array}{l}\text { Bile } \\
\text { acids }\end{array}$ \\
\hline \multicolumn{14}{|l|}{ Studies in mice $(n=30)$} \\
\hline Ryan et al. (37), Ireland & Male C57BL/6 & HFD & $14 / 14$ & $\begin{array}{l}\mathrm{M}(300 \mathrm{mg} / \mathrm{kg}) \text { vs. } \\
\text { non-treatment }\end{array}$ & $12 w$ & Ceca & $\begin{array}{l}16 \mathrm{~S} \text { rRNA V3-V4 } \\
\text { region }\end{array}$ & With vs. without & $\sqrt{ }$ & $\sqrt{ }$ & $\sqrt{ }$ & & \\
\hline Ji et al. (38), China & $\begin{array}{l}\text { Male } \\
\text { C57BL/6J }\end{array}$ & HFD & $5 / 5$ & $\begin{array}{l}\mathrm{M}(300 \mathrm{mg} / \mathrm{kg}) \mathrm{vs} \text {. } \\
\text { non-treatment }\end{array}$ & $3 w$ & Feces & $\begin{array}{l}16 \mathrm{~S} \text { rRNA V4 } \\
\text { region }\end{array}$ & With vs. without & $\sqrt{ }$ & $\sqrt{ }$ & $\sqrt{ }$ & & \\
\hline $\begin{array}{l}\text { Adeshirlarijaney et al. } \\
\text { (41), USA }\end{array}$ & Male C57BL/6 & HFD & 10/NA & $\begin{array}{l}\text { M (300 mg/kg IP) } \\
\text { vs. vehicle }\end{array}$ & $10 w$ & Feces & $\begin{array}{l}16 \mathrm{~S} \text { rRNA V4 } \\
\text { region }\end{array}$ & With vs. without & $\sqrt{ }$ & $\sqrt{ }$ & $\sqrt{ }$ & & \\
\hline \multirow[t]{2}{*}{ Liao et al. (60), China } & Male C57BL/6 & HFD & NA & $\begin{array}{l}\text { A ( } 400 \text { mg } / \mathrm{kg}) \text { vs. Si } \\
(4 \mathrm{~g} / \mathrm{kg}) \text { vs. Sa (300 } \\
\mathrm{mg} / \mathrm{kg}) \text { vs. L (200 } \\
\mu \mathrm{g} / \mathrm{kg}) \text { vs. normal } \\
\text { saline }\end{array}$ & $4 w$ & Feces & $\begin{array}{l}16 S \text { rDNA V3-V4 } \\
\text { region }\end{array}$ & With vs. without (Si) & & $\sqrt{ }$ & $\sqrt{ }$ & $\sqrt{ }$ & \\
\hline & & & & & & & & $\begin{array}{l}\text { With vs. without (A, } \\
\text { Sa, L) }\end{array}$ & & $\sqrt{ }$ & & & \\
\hline $\begin{array}{l}\text { Madsen et al. (65), } \\
\text { Denmark }\end{array}$ & Male C57BL/6 & HFD & $15 / 15$ & $\begin{array}{l}\mathrm{L}(0.4 \mathrm{mg} / \mathrm{kg}) \text { vs. } \\
\text { vehicle }\end{array}$ & $28 d$ & Feces & $\begin{array}{l}16 \mathrm{~S} \text { rDNA V3-V4 } \\
\text { region and } \\
\text { metagenomics }\end{array}$ & Post vs. pre & $\sqrt{ }$ & $\sqrt{ }$ & $\sqrt{ }$ & & \\
\hline Wang et al. (45), Korea & $\begin{array}{l}\text { Male } \\
\text { C57BL/6J }\end{array}$ & HFD & $16 / 8$ & $\begin{array}{l}\mathrm{M}(100 \mathrm{mg} / \mathrm{kg}) \text { vs. } \\
\text { non-treatment }\end{array}$ & $10 w$ & Feces & $\begin{array}{l}\text { 16S rRNA gene } \\
\text { RT-qPCR }\end{array}$ & With vs. without & & $\sqrt{ }$ & $\sqrt{ }$ & & \\
\hline Lee et al. (48), Korea & $\begin{array}{l}\text { Male } \\
\text { C57BL/6N }\end{array}$ & HFD & $6 / 6$ & $\begin{array}{l}\text { M }(250 \mathrm{mg} / \mathrm{kg}) \text { vs. } \\
\text { non-treatment }\end{array}$ & $16 \mathrm{w}$ & Ceca & $\begin{array}{l}16 \mathrm{~S} \text { rRNA V4 } \\
\text { region }\end{array}$ & With vs. without & $\sqrt{ }$ & $\sqrt{ }$ & $\sqrt{ }$ & & \\
\hline Zhou et al. (53), China & $\begin{array}{l}\text { Male } \\
\text { C57BL/6J }\end{array}$ & HFD & NA & $\begin{array}{l}\mathrm{M}(100 \mathrm{mg} / \mathrm{kg}) \text { vs. } \\
\text { non-treatment }\end{array}$ & $4 w$ & Feces & $\begin{array}{l}\text { 16S rRNA gene } \\
\text { RT-qPCR }\end{array}$ & With vs. without & & & $\sqrt{ }$ & & \\
\hline Do et al. (63), Korea & $\begin{array}{l}\text { Male } \\
\text { C57BL/6J }\end{array}$ & HFD & $9 / 10$ & $\begin{array}{l}\text { Vo }(1 \mathrm{mg} / \mathrm{kg}) \mathrm{vs} \text {. } \\
\text { non-treatment }\end{array}$ & $12 w$ & Feces & $\begin{array}{l}16 \mathrm{~S} \text { rRNA V1-V3 } \\
\text { region }\end{array}$ & With vs. without & & & $\sqrt{ }$ & & $\sqrt{ }$ \\
\hline Lee and Ko (55), Korea & $\begin{array}{l}\text { Female } \\
\text { C57BL/6 }\end{array}$ & $\mathrm{ND}, \mathrm{HFD}$ & NA & $\begin{array}{l}\mathrm{M}(300 \mathrm{mg} / \mathrm{kg}) \text { vs. } \\
\text { non-treatment }\end{array}$ & $10 \mathrm{w}$ & Feces & $\begin{array}{l}16 \mathrm{~S} \text { rRNA V1-V3 } \\
\text { region }\end{array}$ & With vs. without & $\sqrt{ }$ & $\sqrt{ }$ & $\sqrt{ }$ & & \\
\hline Shin et al. (56), Korea & C57BL/6 & $\mathrm{ND}, \mathrm{HFD}$ & $12 / 12$ & $\begin{array}{l}\mathrm{M}(300 \mathrm{mg} / \mathrm{kg}) \mathrm{vs} \text {. } \\
\text { non-treatment }\end{array}$ & $6 \mathrm{w}$ & Feces & $\begin{array}{l}\text { 16S rRNA gene } \\
\text { RT-qPCR }\end{array}$ & With vs. without & & $\sqrt{ }$ & $\sqrt{ }$ & & \\
\hline Dong et al. (39), USA & $\mathrm{KC}$ & $\begin{array}{l}\text { Gene knockout } \\
\text { with HFCD }\end{array}$ & $8 / 8$ & $\begin{array}{l}\mathrm{M}(5 \mathrm{mg} / \mathrm{ml} \text { in } \\
\text { drinking water }) \text { vs. } \\
\text { non-treatment }\end{array}$ & $2 \mathrm{~m}$ & $\begin{array}{l}\text { Duodena, ilea, } \\
\text { ceca }\end{array}$ & $\begin{array}{l}16 \mathrm{~S} \text { rRNA V4 } \\
\text { region }\end{array}$ & With vs. without & $\sqrt{ }$ & $\sqrt{ }$ & $\sqrt{ }$ & & \\
\hline $\begin{array}{l}\text { Brandt et al. (40), } \\
\text { Germany }\end{array}$ & $\begin{array}{l}\text { Female } \\
\text { C57BL/6J }\end{array}$ & FFCD & $\begin{array}{c}6-8 / 6- \\
8\end{array}$ & $\begin{array}{l}\mathrm{M}(300 \mathrm{mg} / \mathrm{kg}) \text { vs. } \\
\text { non-treatment }\end{array}$ & $4 d$ & $\begin{array}{l}\text { Proximal small } \\
\text { intestine }\end{array}$ & $\begin{array}{l}16 \mathrm{~S} \text { rRNA V1-V2 } \\
\text { region }\end{array}$ & With vs. without & $\sqrt{ }$ & $\sqrt{ }$ & $\sqrt{ }$ & & \\
\hline \multirow[t]{2}{*}{ Baxter et al. (61), USA } & Male C57BL/6 & HSD, PPD & $25 / 5$ & $\begin{array}{l}\text { A }(25,400 \mathrm{mg} / \mathrm{kg}) \\
\text { vs. non-treatment }\end{array}$ & $2 w$ & Feces & $\begin{array}{l}16 \text { S rRNA V4 } \\
\text { region }\end{array}$ & With vs. without & & $\sqrt{ }$ & $\sqrt{ }$ & $\sqrt{ }$ & \\
\hline & & & & & & & & Post vs. pre & & $\sqrt{ }$ & $\sqrt{ }$ & $\sqrt{ }$ & \\
\hline Kishida et al. (64), Japan & $\begin{array}{l}\text { Male } \\
\text { C57BL/6J }\end{array}$ & HFHSD & $10 / 10$ & $\begin{array}{l}\text { Mi } 0.04 \% \text { in diet vs. } \\
\text { non-treatment }\end{array}$ & $12 w$ & Feces & $\begin{array}{l}16 \mathrm{~S} \text { rRNA V3-V4 } \\
\text { region }\end{array}$ & With vs. without & $\sqrt{ }$ & $\sqrt{ }$ & $\sqrt{ }$ & & \\
\hline $\begin{array}{l}\text { Olivares et al. (74), } \\
\text { Belgium }\end{array}$ & $\begin{array}{l}\text { Male } \\
\text { C57BL/6J }\end{array}$ & WD & 9/9 & $\begin{array}{l}\text { Vi }(0.6 \mathrm{mg} / \mathrm{mL} \text { in } \\
\text { drinking water) vs. } \\
\text { non-treatment }\end{array}$ & $8 w$ & Ceca & $\begin{array}{l}16 \mathrm{~S} \text { rRNA V5-V6 } \\
\text { region }\end{array}$ & With vs. without & $\sqrt{ }$ & $\sqrt{ }$ & $\sqrt{ }$ & $\sqrt{ }$ & \\
\hline Zheng et al. (44), China & $\begin{array}{l}\text { Male } \\
\text { C57BL/6J }\end{array}$ & HFD/STZ & $48 / 8$ & $\begin{array}{l}\text { M }(75,200 \mathrm{mg} / \mathrm{kg}) \\
\text { vs. normal saline }\end{array}$ & $5 w$ & Feces & $\begin{array}{l}16 \mathrm{~S} \text { rRNA V3-V4 } \\
\text { region }\end{array}$ & With vs. without & & $\sqrt{ }$ & $\sqrt{ }$ & & \\
\hline
\end{tabular}


TABLE 3 | Continued

\begin{tabular}{|c|c|c|c|c|c|c|c|c|c|c|c|c|c|}
\hline \multirow[t]{2}{*}{ References, country } & \multirow[t]{2}{*}{ Animals } & \multirow[t]{2}{*}{ Models } & \multirow[t]{2}{*}{$N$} & \multirow{2}{*}{$\begin{array}{l}\text { Treatment and } \\
\text { daily dose }\end{array}$} & \multirow[t]{2}{*}{ Duration } & \multirow[t]{2}{*}{ Specimens } & \multirow{2}{*}{$\begin{array}{l}\text { Analysis } \\
\text { methods }\end{array}$} & \multirow[t]{2}{*}{ Comparison } & \multicolumn{5}{|c|}{ Outcomes } \\
\hline & & & & & & & & & $\alpha$-diversity & $\beta$-diversity & $\begin{array}{l}\text { Taxonomic } \\
\text { composition }\end{array}$ & SCFAs & $\begin{array}{l}\text { Bile } \\
\text { acids }\end{array}$ \\
\hline Wang et al. (70), China & Male ApoE $E^{-/-}$ & $\mathrm{HFD} \pm \mathrm{STZ}$ & $20 / 20 / 20$ & $\begin{array}{l}\mathrm{L}(0.4 \mathrm{mg} / \mathrm{kg}) \text { vs. Sa } \\
(10 \mathrm{mg} / \mathrm{kg}) \text { vs. } \\
\text { non-treatment }\end{array}$ & $8 w$ & Feces & $\begin{array}{l}16 \mathrm{~S} \text { rRNA V1-V3 } \\
\text { region }\end{array}$ & $\begin{array}{l}\text { With vs. without (L, } \\
\text { Sa) }\end{array}$ & $\sqrt{ }$ & $\sqrt{ }$ & $\sqrt{ }$ & & \\
\hline Xue et al. (36), China & $\begin{array}{l}\text { Female } \\
\text { C57BL/6J }\end{array}$ & DHEA+HFD & $10 / 10$ & $\begin{array}{l}\text { M }(1.9 \mathrm{~g} / \mathrm{kg}) \text { vs. } \\
\text { normal saline }\end{array}$ & $21 d$ & Feces & $\begin{array}{l}\text { 16S rDNA V3-V4 } \\
\text { region }\end{array}$ & With vs. without & & $\sqrt{ }$ & $\sqrt{ }$ & & \\
\hline Moreira et al. (68), Brazil & $\begin{array}{l}\text { Male } \\
\text { C57BL/6J and } \\
\text { female ob/ob }\end{array}$ & $\begin{array}{l}\text { ND, HFD, gene } \\
\text { knockout }\end{array}$ & $\begin{array}{c}24- \\
48 / 24- \\
48\end{array}$ & $\begin{array}{l}\mathrm{L}(400 \mu \mathrm{g} / \mathrm{kg}) \mathrm{vs} \text {. } \\
\text { normal saline }\end{array}$ & $15 d$ & Feces & $\begin{array}{l}16 \mathrm{~S} \text { rRNA V3-V4 } \\
\text { region }\end{array}$ & With vs. without & $\sqrt{ }$ & & $\sqrt{ }$ & & \\
\hline Ma et al. (46), China & C57BL/6 & ND & $10 / 9$ & $\begin{array}{l}\text { M (300 mg/kg) vs. } \\
\text { normal saline }\end{array}$ & $30 \mathrm{~d}$ & Feces & $16 \mathrm{~S}$ rRNA & With vs. without & $\sqrt{ }$ & $\sqrt{ }$ & $\sqrt{ }$ & & \\
\hline Xu et al. (62), China & Male ICR & ND & $5 / 5 / 5$ & $\begin{array}{l}\text { A }(4 \mathrm{mg} / \mathrm{kg}) \text { vs. Vo } \\
(0.008 \mathrm{mg} / \mathrm{kg}) \text { vs. } \\
\text { non-treatment }\end{array}$ & $2 w$ & Intestine & $\begin{array}{l}\text { 16S rRNA V4 } \\
\text { region }\end{array}$ & With vs. without & & & $\sqrt{ }$ & $\sqrt{ }$ & \\
\hline Zhang et al. (35), China & $\begin{array}{l}\text { BKSLeprdb } \\
(d b / d b)\end{array}$ & Gene knockout & $5 / 5$ & $\begin{array}{l}\mathrm{M}(113.75 \mathrm{mg} / \mathrm{kg}) \\
\text { vs. non-treatment }\end{array}$ & $11 w$ & Feces & $\begin{array}{l}16 \mathrm{~S} \text { rRNA V3-V4 } \\
\text { region }\end{array}$ & With vs. without & $\sqrt{ }$ & $\sqrt{ }$ & $\sqrt{ }$ & $\sqrt{ }$ & \\
\hline Lee et al. (76), USA & $\begin{array}{l}\text { C57BLKS/J- } \\
\text { leprdb/leprdb } \\
(d b / d b)\end{array}$ & Gene knockout & $12 / 12$ & $\begin{array}{l}\mathrm{D}(60 \mathrm{mg} / \mathrm{kg} \text { in diet) } \\
\text { vs. non-treatment }\end{array}$ & $8 w$ & Feces & $\begin{array}{l}\text { 16S rRNA V4 } \\
\text { region }\end{array}$ & With vs. without & $\sqrt{ }$ & $\sqrt{ }$ & $\sqrt{ }$ & & \\
\hline Li et al. (75), China & $\begin{array}{l}\text { Male ICR } \\
\text { MafA-deficient }\end{array}$ & Gene knockout & $8 / 8$ & $\begin{array}{l}\mathrm{D}(1.0 \mathrm{mg} / \mathrm{kg}) \mathrm{vs} . \\
\text { normal saline }\end{array}$ & $6 \mathrm{w}$ & $\begin{array}{l}\text { Intestine and } \\
\text { feces }\end{array}$ & $\begin{array}{l}16 \mathrm{~S} \text { rRNA V3-V4 } \\
\text { region }\end{array}$ & With vs. without & $\sqrt{ }$ & $\sqrt{ }$ & $\sqrt{ }$ & $\sqrt{ }$ & \\
\hline Li et al. (78), China & Female KKAy & Gene knockout & $6 / 6$ & P vs. distilled water & NA & Feces & $16 \mathrm{~S}$ rDNA DGGE & With vs. without & $\sqrt{ }$ & $\sqrt{ }$ & $\sqrt{ }$ & & \\
\hline Wang et al. (79), China & KKAy & Gene knockout & $7 / 6$ & $\begin{array}{l}\mathrm{R}(2 \mathrm{mg} / \mathrm{kg}) \text { vs. } \\
\text { distilled water }\end{array}$ & $8 w$ & Intestine & Cultivation & With vs. without & & & $\sqrt{ }$ & & \\
\hline Smith et al. (59), USA & $\begin{array}{l}\text { Offsprings of } \\
\text { female CByB6 } \\
\mathrm{mF} 1 / \mathrm{J} \text { and } \\
\text { male } \\
\text { C3D2F1/J }\end{array}$ & ND & $71 / 72$ & $\begin{array}{l}\text { A ( } 1,000 \text { ppm with } \\
\text { diet) vs. } \\
\text { non-treatment }\end{array}$ & $17-25 \mathrm{~m}$ & Feces & $\begin{array}{l}16 \mathrm{~S} \text { rRNA V4 } \\
\text { region }\end{array}$ & With vs. without & $\sqrt{ }$ & $\sqrt{ }$ & $\sqrt{ }$ & $\sqrt{ }$ & \\
\hline $\begin{array}{l}\text { Salomäki-Myftari et al. } \\
\text { (52), Finland }\end{array}$ & $\begin{array}{l}\text { Offsprings of } \\
\text { homozygous } \\
\text { OE-NPY }\end{array}$ & Gene knockout & NA & $\begin{array}{l}\text { M (300 mg/ } / \mathrm{kg} \text { ) vs. } \\
\text { vehicle (for dams) }\end{array}$ & $18 d$ & Feces & $\begin{array}{l}16 \mathrm{~S} \text { rRNA V4-V5 } \\
\text { region }\end{array}$ & With vs. without & & $\sqrt{ }$ & $\sqrt{ }$ & & \\
\hline Mishima et al. (77), Japan & Male C57BL/6 & $\begin{array}{l}\text { Adenine } \\
\text { induced renal } \\
\text { failure }\end{array}$ & $8 / 8$ & $\begin{array}{l}\mathrm{C}(10 \mathrm{mg} / \mathrm{kg}) \text { vs. } \\
\text { vehicle }\end{array}$ & $2 \mathrm{w}$ & Ceca & $\begin{array}{l}16 \mathrm{~S} \text { rRNA V1-V2 } \\
\text { region }\end{array}$ & With vs. without & & $\sqrt{ }$ & $\sqrt{ }$ & $\sqrt{ }$ & \\
\hline \multicolumn{14}{|l|}{ Studies in rats $(n=17)$} \\
\hline Bauer et al. (43), Canada & Male SD & HFD & $6 / 6$ & $\begin{array}{l}\text { M (200 mg/kg) vs. } \\
\text { normal saline }\end{array}$ & $1 \mathrm{~d}$ & Lumina & $\begin{array}{l}16 \mathrm{~S} \text { rRNA V3 } \\
\text { region }\end{array}$ & With vs. without & $\sqrt{ }$ & $\sqrt{ }$ & $\sqrt{ }$ & & \\
\hline Zhang et al. (54), China & Male W & HFD & $10 / 10$ & $\begin{array}{l}\text { M }(200 \mathrm{mg} / \mathrm{kg}) \text { vs. } \\
\text { vehicle }\end{array}$ & $8 w$ & Feces & $\begin{array}{l}16 \mathrm{~S} \text { rRNA V3 } \\
\text { region }\end{array}$ & With vs. without & $\sqrt{ }$ & $\sqrt{ }$ & $\sqrt{ }$ & & \\
\hline Pyra et al. (57), Canada & Male SD & HFHSD & $20 / 10$ & $\begin{array}{l}\mathrm{M}(300 \mathrm{mg} / \mathrm{kg}) \mathrm{vs} . \\
\text { non-treatment }\end{array}$ & $7 \mathrm{w}$ & Ceca & $\begin{array}{l}\text { DNA gene } \\
\text { RT-qPCR }\end{array}$ & With vs. without & & & $\sqrt{ }$ & & \\
\hline $\begin{array}{l}\text { Dennison et al. (72), } \\
\text { Canada }\end{array}$ & Female SD & HFHSD & $\begin{array}{c}11- \\
13 / 11- \\
13\end{array}$ & $\begin{array}{l}\mathrm{Si}(10 \mathrm{mg} / \mathrm{kg}) \text { vs. } \\
\text { non-treatment }\end{array}$ & $12 \mathrm{w}$ & Feces & $\begin{array}{l}\text { 16S rRNA gene } \\
\text { RT-qPCR }\end{array}$ & With vs. without & & & $\sqrt{ }$ & & \\
\hline Liu et al. (47), China & Male W & HFD/STZ & $10 / 10$ & $\begin{array}{l}\mathrm{M}(200 \mathrm{mg} / \mathrm{kg}) \mathrm{vs} \text {. } \\
\text { non-treatment }\end{array}$ & $4 \mathrm{w}$ & Colon & $\begin{array}{l}16 S \text { rRNA V3-V4 } \\
\text { region }\end{array}$ & With vs. without & $\sqrt{ }$ & $\sqrt{ }$ & $\sqrt{ }$ & & \\
\hline
\end{tabular}


TABLE 3 | Continued

References, country

Animals

Models

N Treatment and

Duration Specimens

Analysis

Compariso

Outcomes daily dose

$\alpha$-diversity $\beta$-diversity $\quad$ Taxonomic

SCFAs Bile composition

Xu M et al. (42), China SD

HFHSD/STZ

10/10 M $(1.8 \mathrm{~g} / \mathrm{kg})$ vs non-M

Zhang et al. (69), China Male SD

HFD/STZ

6/6 L $(0.4 \mathrm{mg} / \mathrm{kg}) \mathrm{vs}$.

normal saline

Zhang et al. (6), China Male SD HFD/STZ

Yan et al. (73), China $\quad$ Male SD

HFHC/STZ

$12 / 6 \quad$ Vi $(0.01,0.02 \mathrm{~g} / \mathrm{kg}$ vs. vehicle

Yuan et al. (67), China $\quad$ Male SD

STZ

non-treatment

Zhang et al. (5), China Male ZDF

Gene knockout $8 / 8 / 8 / 8$ non-L

( $0.6 \mathrm{mg} / \mathrm{kg})$ vs.

4w Feces

$16 \mathrm{~S}$ rDNA gene

With vs. without

$\sqrt{ }$

QPCR

16S rRNA V3-V4 With vs, without

region

16S rRNA V3-V4 With vs. without

region

16S rRNA V1-V3 With vs. without

region

16S rRNA V3 With vs. without

region

A $(32.27 \mathrm{mg} / \mathrm{kg})$ vs
M $(215.15 \mathrm{mg} / \mathrm{kg})$
vs. Si $(10.76 \mathrm{mg} / \mathrm{kg})$

vs. normal saline

Shin et al. (49), Korea

Male OLETF

Gene knockout

M (100 mg/kg) vs.

water

Wang et al. (50), Korea

Male OLETF

Gene knockout

M $(100 \mathrm{mg} / \mathrm{kg})$ vs.

distilled water

Han et al. (51), Korea

Male OLETF

Gene knockout

M (100 mg/kg) vs.

distilled water

Zhao et al. (58), China

Male GK

Gene knockout

6/6 A (50 mg/kg) vs.

normal saline

Zhao et al. (66), China

Male $W$ and

GK

HFD, gene $\quad 16 / 16 \quad L(400 \mu \mathrm{g} / \mathrm{kg}) \mathrm{vs}$.

knockout

normal saline

Kaya et al. (71), Japan Male OLETF

and PS

vehicle

16S rRNA V3-V4 A vs. M

region

A vs. S

With vs. without (A)

With vs. without (M,

Si)

16S rRNA V1-V3 With vs. withou

region

16S rRNA V3 With vs. without

region

16S rRNA V1-V2 With vs. without

region

16S rRNA V3-V4 With vs. without

region

16 S rRNA V3-V4 With vs. without

region

16S rRNA V4

region

A, acarbose; An, anagliptin; C, canagliflozin; D, dapagliflozin; G, glipizide; Mi, miglitol; M, metformin; L, liraglutide; P, pioglitazone; R, rosiglitazone; Si, sitagliptin; Sa, saxagliptin; Vo, voglibose; Vi, vildagliptin; HFD, high-fat diet; ND, normal-chow diet; HFHSD, high-fat high-sucrose diet; HFHCD, high-fat high-carbohydrate diet; DHEA, trans-dehydroandrosterone; HFCD, high-fat high-calories diet; FFCD, fat-, fructose-, and cholesterol-rich diet; HSD, high-starch diet; PPD, plant plolysaccharide diet; WD, Western diet; STZ, streptozocin intraperitoneal injection; DHEA, trans-dehydroandrosterone; PS, pocrine serum intraperitoneal injection; IP, Intraperitoneal injection; SD rats, Sprague-Dawley rats; ZDF rats, Male Zucker diabetic fatty rats, induced by leptin receptor gene knockout; KC mice, LSL-KrasG12D/+ and p48-Cre+/- mice, induced by LSL-KRASG12D and Cre alleles knockout; OLETF rats, Otsuka Long-Evans Tokushima Fatty rats, induced by spontaneous CCK receptor knockout: OE-NPY mice, homozygous transgenic OE-NPY mice, induced by transgenic mice overexpressing Neuropeptide Y under dopamine- $\beta$-hydroxylase promoter; $W$ rats, Wistar rats; GK rats, Goto-Kakizaki rats, induced by polygenic Wistar substrain; ob/ob mice, mice model induced by Lep pob gene knockout; ICR MafA-deficient mice, model induced by targeted disruption of the mafA gene in ICR mice; db/db mice, model induced by mutation in the leptin receptor gene in mice; KKAy mice, induced by transfer the yellow obese gene (AV) into KK mice; RT-qPCR, Real-time quantitative polymerase chain reaction; DGGE, denaturing gradient gel electrophoresis; $m$, months; $w$, weeks; $d$, days; NA, not available; vs., versus. 
TABLE 4 | Effects of anti-hyperglycemic drugs on specific taxa in human gut microbiota, categorized by the target research populations ${ }^{a}$.

\begin{tabular}{|c|c|c|c|c|c|c|c|c|}
\hline Specific taxa & Phylum & $N^{b}$ & $N / N^{c}$ & Healthy & Obese & Pre-diabetic & Newly T2D & $\begin{array}{c}\text { Prevalent } \\
\text { T2D }\end{array}$ \\
\hline G_Alistipes & Bacteroidetes & 2 & $151 / 151$ & & & & $\downarrow A(32), M(20)$ & \\
\hline G_Bacteroides & Bacteroidetes & 5 & $233 / 233$ & & $\leftrightarrow \mathrm{M}(19)$ & $\downarrow A(7)$ & $\downarrow A(32), M(20,24)$ & \\
\hline S_Bacteroides dorei & Bacteroidetes & 2 & $73 / 73$ & & & & $\downarrow A(32), M(24)$ & \\
\hline S_Bacteroides finegoldii & Bacteroidetes & 2 & $73 / 73$ & & & & $\downarrow \mathrm{A}(32), \mathrm{M}(24)$ & \\
\hline S_Bacteroides intestinalis & Bacteroidetes & 2 & $73 / 73$ & & & & $\downarrow \mathrm{A}(32), \mathrm{M}(24)$ & \\
\hline S_Bacteroides stercoris & Bacteroidetes & 2 & $73 / 73$ & & & & $\downarrow \mathrm{A}(32), \mathrm{M}(24)$ & \\
\hline S_Bacteroides thetaiotaomicron & Bacteroidetes & 2 & $73 / 73$ & & & & $\downarrow \mathrm{A}(32), \mathrm{M}(24)$ & \\
\hline S_Bacteroides uniformis & Bacteroidetes & 2 & $73 / 73$ & & & & $\downarrow \mathrm{A}(32), \mathrm{M}(24)$ & \\
\hline S_Bacteroides vulgatus & Bacteroidetes & 2 & $73 / 73$ & & & & $\downarrow \mathrm{A}(32), \mathrm{M}(24)$ & \\
\hline G_Bifidobacterium & Actinobacteria & 2 & $73 / 73$ & & & & $\uparrow A(32), M(21)$ & \\
\hline S_Bifidobacterium adolescentis & Actinobacteria & 2 & $73 / 73$ & & & & $\uparrow A(32), M(21)$ & \\
\hline G_Clostridium & Firmicutes & 2 & $76 / 76$ & $\downarrow M(22)$ & & & $\downarrow A(32)$ & \\
\hline S_Clostridium leptum & Firmicutes & 2 & $71 / 84$ & & & & $\downarrow A(32)$ & $\downarrow M(31)$ \\
\hline F_Lachnospiraceae & Firmicutes & 2 & $140 / 140$ & & & $\downarrow A(7)$ & $\uparrow \mathrm{M}(20)$ & \\
\hline G_Lactobacillus & Firmicutes & 3 & $111 / 111$ & & $\leftrightarrow \mathrm{M}(19)$ & $\uparrow A(7)$ & $\uparrow A(32)$ & \\
\hline S_Lactobacillus gasseri & Firmicutes & 2 & $73 / 73$ & & & & $\uparrow A(32), M(21)$ & \\
\hline G_Megasphaera & Firmicutes & 2 & $54 / 54$ & & & $\uparrow A(7)$ & & $\uparrow \mathrm{M}(29)$ \\
\hline S_Pseudoflavonifractor capillosus & Firmicutes & 2 & $73 / 73$ & & & & $\downarrow A(32), M(21)$ & \\
\hline S_Ruminococcus sp. 5_1_39BFAA & Firmicutes & 2 & $73 / 73$ & & & & $\downarrow \mathrm{A}(32), \uparrow \mathrm{M}(21)$ & \\
\hline
\end{tabular}

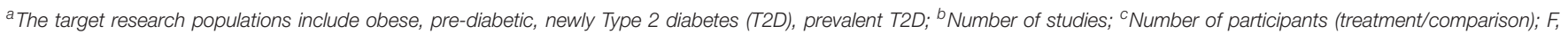
family; G, genus; S, species; M, metformin; A, acarbose; $\uparrow$, significant increase; $\downarrow$, significant decrease; $\leftrightarrow$, no significant difference.

This decrease was also noted in another two studies, in which liraglutide was given to in rat models induced by diets and gene knockout $(66,69)$.

Responses of the phylum Bacteroidetes to different antihyperglycemic agents were investigated in 15 rodent studies. No difference was noted in studies treated with acarbose $(5,58)$. Five out of the seven studies on the genus Bacteroides after using metformin $(37,42,48,54,56)$ revealed increased abundance of this genus in mice and rats.

Among 15 rodent studies (5, 6, 36, 37, 41, 47, 48, 54, 56, 58, $66,69,70,73,75)$ on the phylum Firmicutes, the results were inconclusive among those treated with metformin $(5,36,37$, $41,47,48,54,56)$, acarbose $(5,58)$, liraglutide $(66,69,70)$, and sitagliptin $(5,73)$. The genus Lactobacillus was the focus of 13 studies $(5,35,41-43,47,53,54,58,62,66,70,73)$. Six out of the eight studies treated with metformin $(5,35,43,53,54,62)$ saw an increase in this genus in mice and rats, while the results in studies treated with acarbose $(5,58,62)$, liraglutide $(66,70)$, and sitagliptin $(5,73)$ were inconclusive.

With respect to other phyla, there was a trend of decrease in the phylum Proteobacteria in mice treated with metformin and liraglutide, while Verrucomicrobia and Tenericutes increased after treated with metformin and liraglutide, respectively. However, results for the phyla Actinobacteria, Cyanobacteria, Elusimicrobia, and Fusobacteria were conflicting. The genus Akkermanisa (phylum Verrucomicrobia) increased in eight studies treated with metformin using dietary or genetic models $(35,37,38,41,44,48,54,56)$. Three of the four studies with metformin $(41,53,55)$ reported an increase in the species $A$. muciniphila, and another two studies reported a similar increase in this species after treating with liraglutide $(65,68)$.

\section{Diversity}

Ten human studies treated with metformin $(19,20,22-25,27-$ $30)$ and two studies treated with acarbose $(7,32)$ have provided the results of $\alpha$-diversity. The results from those metformin studies, however, were conflicting, while both acarbose studies reported a decrease in the $\alpha$-diversity among pre-diabetic and T2D patients. $\beta$-diversity was assessed in ten studies treated with metformin (19-22, 24, 25, 27-29, 34), of which six studies (20$22,24,27,34)$ revealed a significant difference after the treatment in healthy participants and T2D patients. This difference was also noted in pre-diabetic patients treated with acarbose (32), and T2D patients treated with liraglutide (34) (Table 8).

Similar results with metformin and acarbose were reported in mouse studies. The effects of metformin on $\alpha$-diversity were conflicting across different models, while the $\alpha$-diversity decreased consistently in three studies treated with acarbose (5, 58, 59). Moreover, the results were inconsistent among those studies treated with liraglutide, sitagliptin, vildagliptin, and saxagliptin. In terms of $\beta$-diversity, there was higher cumulative evidence of significant difference after using metformin $(5,36-$ $39,41,43-50,54-56)$, and similar results were consistently reported among those studies treated with acarbose (5, 58$61)$, liraglutide $(60,65,66,69,70)$, sitagliptin $(5,60,73)$, and 


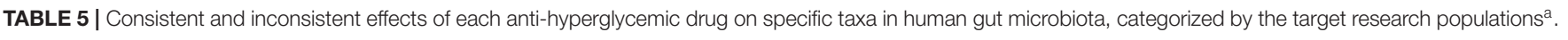

\begin{tabular}{lllllll}
\hline Drug/specific taxa & Phylum & $N^{b}$ & $N / N^{c}$ & Healthy & Obese & Pre-diabetic \\
T2D
\end{tabular}

\begin{tabular}{|c|c|c|c|c|c|c|c|c|}
\hline \multicolumn{9}{|l|}{ METFORMIN } \\
\hline \multicolumn{9}{|l|}{ Consistent results } \\
\hline G_Fusobacterium & Fusobacteria & 2 & $151 / 126$ & & & & $\uparrow(20,27)$ & \\
\hline S_Akkermansia muciniphila & Verrucomicrobia & 2 & $36 / 36$ & & & & $\uparrow(21)$ & $\uparrow(29)$ \\
\hline G_Escherichia & Proteobacteria & 6 & $243 / 202$ & $\begin{array}{c}\uparrow \\
(22,25)\end{array}$ & $\uparrow(19)$ & & $\uparrow(20,21)$ & $\uparrow(30)$ \\
\hline G_Shigella & Proteobacteria & 4 & $163 / 163$ & $\begin{array}{c}\uparrow \\
(22,25)\end{array}$ & $\uparrow(19)$ & & $\uparrow(20)$ & \\
\hline S_Citrobacter koseri & Proteobacteria & 2 & $42 / 55$ & & & & $\uparrow(21)$ & $\uparrow(31)$ \\
\hline S_Citrobacter rodentium & Proteobacteria & 2 & $42 / 55$ & & & & $\uparrow(21)$ & $\uparrow(31)$ \\
\hline S_Enterobacter cloacae & Proteobacteria & 2 & $42 / 55$ & & & & $\uparrow(21)$ & $\uparrow(31)$ \\
\hline S_Escherichia coli & Proteobacteria & 2 & $42 / 55$ & & & & $\uparrow(21)$ & $\uparrow(31)$ \\
\hline S_Klebsiella pneumonia & Proteobacteria & 2 & $42 / 55$ & & & & $\uparrow(21)$ & $\uparrow(31)$ \\
\hline S_Erwinia amylovora & Proteobacteria & 2 & $42 / 55$ & & & & $\uparrow(21)$ & $\uparrow(31)$ \\
\hline S_Pectobacterium wasabiae & Proteobacteria & 2 & $42 / 55$ & & & & $\uparrow(21)$ & $\uparrow(31)$ \\
\hline S_Dickeya dadantii & Proteobacteria & 2 & $42 / 55$ & & & & $\uparrow(21)$ & $\uparrow(31)$ \\
\hline S_Clostridium bartlettii & Firmicutes & 2 & $42 / 55$ & & & & $\downarrow(21)$ & $\downarrow(31)$ \\
\hline S_Clostridium botulinum & Firmicutes & 2 & $42 / 55$ & & & & $\downarrow(21)$ & $\downarrow(31)$ \\
\hline \multicolumn{9}{|l|}{ Inconsitent results } \\
\hline G_Bacteroides & Bacteroidetes & 3 & $142 / 142$ & & $\leftrightarrow(19)$ & & $\downarrow(20,24)$ & \\
\hline G_Prevotella & Bacteroidetes & 2 & $34 / 34$ & & $\leftrightarrow(19)$ & & & $\uparrow(29)$ \\
\hline G_Blautia & Firmicutes & 3 & $138 / 138$ & $\leftrightarrow(25)$ & $\leftrightarrow(19)$ & & $\uparrow(20)$ & \\
\hline G_Intestinibacter & Firmicutes & 4 & $125 / 84$ & $\downarrow(22)$ & $\leftrightarrow(19)$ & & $\downarrow(21)$ & $\downarrow(30)$ \\
\hline F_Enterobacteriaceae & Proteobacteria & 2 & $69 / 44$ & $\uparrow(25)$ & & & & $\downarrow(27)$ \\
\hline \multicolumn{9}{|l|}{ Acarbose } \\
\hline \multicolumn{9}{|l|}{ Consistent results } \\
\hline G_Bacteroides & Bacteroidetes & 2 & $91 / 91$ & & & $\downarrow(7)$ & $\downarrow(32)$ & \\
\hline G_Lactobacillus & Firmicutes & 2 & $91 / 91$ & & & $\uparrow(7)$ & $\uparrow(32)$ & \\
\hline S_Bidifobacterium Iongum & Actinobacteria & 2 & $110 / 110$ & & & & $\uparrow(32)$ & $\uparrow(33)$ \\
\hline
\end{tabular}

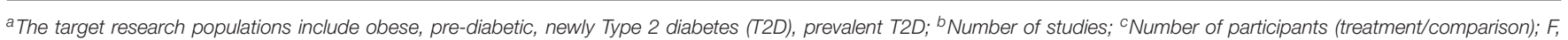
family; G, genus; S, species; $\uparrow$, significant increase; $\downarrow$, significant decrease; $\leftrightarrow$, no significant difference.

vildagliptin $(6,74)$ across different mouse models. Evidence for the effects of other drugs was limited (Table 9).

\section{Short-Chain Fatty Acids (SCFAs)}

In human studies, changes in the levels of three main SCFAs (acetate, propionate, and butyrate) in feces and sera were reported in three studies treated with metformin $(19,21,23)$ (Supplementary Table 3). Wu et al. (21) found that the levels of fecal butyrate and propionate increased in T2D male patients. However, no difference in fecal levels of these two SCFAs was noted among obese women in Ejtahed's study (19). In contrast, fecal acetate levels decreased in obese women (19) did not change among T2D patients in Wu's study (21). Huang et al. (23) reported that the serum levels of all three SCFAs remained unchanged after treating with metformin in T2D patients.

In mouse studies, fecal levels of SCFAs after metformin interventions were assessed in $d b / d b$ mice (35) and OLETF rats (50) (Supplementary Table 3). It was found that levels of acetate and butyrate increased, but propionate levels remained unchanged. The effects of acarbose on these SCFAs levels were also assessed in dietary models $(59,61,62)$. These studies showed consistent results of increased levels of butyrate in feces and ceca, but the levels of acetate and propionate varied in a dietdependent manner.

\section{Bile Acids}

In human studies, three clinical trials treated with metformin (21, $24,26)$ and one randomized trial with acarbose and glipizide (32) were carried out in T2D patients to assess the respective effects on the fecal and serum levels of bile acids (Supplementary Table 4). Regarding metformin, one study (24) showed increases in the fecal level of conjugated secondary bile acids, while no difference was reported in the other two studies $(21,26)$. Two of these three studies $(21,24)$ reported increases in the blood level of 
TABLE 6 | Effects of anti-hyperglycemic drugs on gut microbiota in mouse models, categorized by the treated, individual anti-hyperglycemic drug.

\begin{tabular}{|c|c|c|c|c|c|c|c|c|c|c|c|c|c|}
\hline Specific taxon & $N^{\mathrm{a}}$ & $\mathbf{M}$ & A & Mi & Vo & $\mathbf{L}$ & An & Sa & $\mathrm{Si}$ & Vi & C & D & $\mathbf{R}$ \\
\hline Firmicutes/bacteroidetes & 9 & $\downarrow(2)$ & $\leftrightarrow$ & & $\downarrow$ & $\downarrow(2)$ & $\downarrow$ & & & $\downarrow$ & & $\downarrow$ & \\
\hline \multicolumn{14}{|l|}{ Phylum Bacteroidetes } \\
\hline P_Bacteriodetes & 15 & $\leftrightarrow(3), \downarrow, \uparrow(5)$ & $\leftrightarrow(2)$ & & & $\downarrow, \uparrow(2)$ & & $\downarrow$ & $\leftrightarrow, \uparrow(2)$ & $\uparrow$ & & $\uparrow$ & \\
\hline C_Bacteroidia & 3 & $\uparrow$ & & & & $\downarrow, \uparrow$ & & & & & & & \\
\hline F_Bacteroidaceae & 5 & $\uparrow$ & $\uparrow$ & & & $\downarrow$ & & & $\uparrow$ & $\uparrow$ & & & \\
\hline S_Bacteroides acidifaciens & 2 & & & & & $\uparrow$ & & & & $\uparrow$ & & $\uparrow$ & \\
\hline G_Butyricimonas & 3 & $\uparrow(2)$ & & & & $\uparrow$ & & & & & & & \\
\hline F_Porphyromonadaceae & 4 & $\uparrow(2)$ & & & & $\downarrow, \uparrow$ & & & & & & & \\
\hline G_Odoribacter & 4 & $\leftrightarrow, \downarrow(2)$ & $\leftrightarrow$ & & & & & & & & & & \\
\hline G_Parabacteroides & 8 & $\downarrow, \uparrow(4)$ & $\leftrightarrow$ & & & $\downarrow$ & & & $\uparrow$ & & & & \\
\hline F_Rikenellaceae & 3 & $\uparrow(2)$ & & $\uparrow$ & & & & & & & & & \\
\hline G_Alistipes & 3 & $\uparrow$ & $\uparrow$ & & $\uparrow$ & & & & & & & $\uparrow$ & \\
\hline F_S24-7 & 3 & & $\downarrow, \uparrow$ & & & & & & $\uparrow$ & & & & \\
\hline \multicolumn{14}{|l|}{ Phylum Firmicutes } \\
\hline P_Firmicutes & 15 & $\leftrightarrow(4), \downarrow(3), \uparrow(2)$ & $\leftrightarrow, \uparrow$ & & & $\leftrightarrow, \downarrow, \uparrow$ & & $\uparrow$ & $\downarrow, \uparrow$ & $\downarrow$ & & $\uparrow$ & \\
\hline G_Turicibacter & 5 & $\leftrightarrow, \downarrow, \uparrow$ & $\leftrightarrow$ & & & $\uparrow$ & & $\uparrow$ & & & & & \\
\hline O_Lactobacillales & 2 & $\downarrow$ & & & & $\uparrow$ & & & & & & & \\
\hline G_Enterococcus & 7 & $\leftrightarrow, \downarrow(3), \uparrow$ & & & & & & & & & & $\downarrow, \uparrow$ & \\
\hline F_Lactobacillaceae & 5 & $\downarrow, \uparrow$ & $\downarrow(2)$ & & & $\uparrow$ & & & & & & & \\
\hline G_Lactobacillus & 13 & $\downarrow(2), \uparrow(6)$ & $\leftrightarrow, \downarrow, \uparrow$ & & & $\downarrow, \uparrow$ & & $\uparrow$ & $\downarrow, \uparrow$ & & & & \\
\hline F_Clostridiales_vadinBB60_g & 2 & & & & & $\uparrow$ & & & $\downarrow$ & & & & \\
\hline F_Christensenellaceae & 3 & $\uparrow$ & & & & $\downarrow$ & & & & $\downarrow$ & & & \\
\hline G_Christensenellaceae $R \_7 \_g$ & 3 & & $\leftrightarrow$ & & & $\leftrightarrow, \downarrow$ & & & & $\downarrow$ & & & \\
\hline G_Candidatus Arthromitus & 3 & $\leftrightarrow$ & $\leftrightarrow$ & & & $\downarrow$ & & & & & & & \\
\hline G_Clostridium & 3 & $\downarrow$ & & & & $\uparrow$ & & & $\leftrightarrow$ & & & & \\
\hline G_Ruminiclostridium & 2 & & $\leftrightarrow$ & & & & & & $\downarrow$ & & & & \\
\hline G_Ruminiclostridium 6 & 2 & & $\downarrow$ & & $\downarrow$ & $\downarrow$ & & & & $\downarrow$ & & & \\
\hline G_Ruminiclostridium 9 & 3 & & $\leftrightarrow$ & & & $\leftrightarrow$ & & & $\downarrow$ & & & & \\
\hline F_Ruminococcaceae & 6 & $\uparrow$ & $\downarrow$ & & & $\leftrightarrow, \uparrow$ & & & $\downarrow$ & $\downarrow$ & & & \\
\hline G_Ruminococcaceae_UCG_005 & 1 & & $\downarrow$ & & $\downarrow$ & & & & & & & & \\
\hline G_Ruminococcus & 5 & $\downarrow(2), \uparrow(2)$ & & & & & & & $\uparrow$ & & & & \\
\hline G_Ruminococcus 2 & 2 & & $\downarrow, \uparrow$ & & $\uparrow$ & & & & & & & & \\
\hline F_Lachnospiraceae & 5 & $\downarrow$ & $\leftrightarrow$ & & & $\leftrightarrow, \downarrow$ & & & $\downarrow$ & & & & \\
\hline G_Blautia & 8 & $\downarrow, \uparrow$ & $\uparrow$ & & $\uparrow$ & $\uparrow(2)$ & & & $\downarrow(2)$ & & & $\uparrow$ & \\
\hline G_Lachnoclostridium & 3 & $\downarrow$ & $\leftrightarrow$ & & & $\uparrow$ & & & & & & & \\
\hline G_Lachnospiraceae_nk4a136_g & 2 & & & & & $\leftrightarrow$ & & & & & & $\uparrow$ & \\
\hline G_Marvinbryantia & 2 & & $\leftrightarrow$ & & & $\downarrow$ & & & & & & & \\
\hline
\end{tabular}


TABLE 6 | Continued

\begin{tabular}{|c|c|c|c|c|c|c|c|c|c|c|c|c|c|}
\hline Specific taxon & $N^{a}$ & $\mathbf{M}$ & A & Mi & Vo & $\mathbf{L}$ & An & Sa & Si & Vi & C & D & $\mathbf{R}$ \\
\hline G_Roseburia & 7 & $\leftrightarrow, \downarrow$ & $\leftrightarrow$ & & & $\leftrightarrow, \downarrow$ & & & $\leftrightarrow, \downarrow$ & & & & \\
\hline G_Peptococcus & 2 & $\downarrow$ & $\leftrightarrow$ & & & & & & & & & & \\
\hline G_Romboustia & 2 & $\downarrow$ & & & & $\downarrow$ & & & & & & & \\
\hline G_Anaerotruncus & 6 & $\leftrightarrow, \downarrow$ & $\leftrightarrow$ & & & $\downarrow$ & & & $\downarrow$ & $\downarrow$ & & & \\
\hline G_Flavonifractor & 2 & $\downarrow$ & & & & $\uparrow$ & & & & & & & \\
\hline G_Oscillospira & 6 & $\downarrow, \uparrow$ & & & & $\uparrow$ & & & & $\downarrow$ & $\downarrow$ & $\downarrow$ & \\
\hline F_Dehalobacteriaceae & 3 & $\downarrow, \uparrow$ & & $\uparrow$ & & & & & & & & & \\
\hline F_Erysipelotrichaceae & 5 & $\uparrow$ & $\leftrightarrow, \uparrow$ & $\downarrow$ & & $\uparrow$ & & & & & & & \\
\hline O_Erysipelotrichales & 2 & $\uparrow$ & & & & $\uparrow$ & & & & & & & \\
\hline \multicolumn{14}{|l|}{ Phylum Actinobacteria } \\
\hline P_Actinobacteria & 5 & $\leftrightarrow$ & $\uparrow$ & & & $\leftrightarrow, \downarrow$ & & & & & $\downarrow$ & & \\
\hline F_Bifidobacteriaceae & 2 & $\downarrow$ & $\uparrow$ & & & & & & & & & & \\
\hline G_Bifidobacterium & 8 & $\leftrightarrow(2), \uparrow, \downarrow$ & $\uparrow$ & & & $\uparrow$ & & & $\uparrow$ & & $\downarrow$ & & \\
\hline S_Bifidobacterium spp. & 2 & $\downarrow$ & & & & & & & & $\leftrightarrow$ & & & \\
\hline G_Corynebacterium 1 & 2 & & $\leftrightarrow$ & & & & & & $\uparrow$ & & & & \\
\hline G_Enterorhabdus & 2 & & $\leftrightarrow$ & & & $\downarrow$ & & & & & & & \\
\hline \multicolumn{14}{|l|}{ Phylum Cyanobacteria } \\
\hline P_Cyanobacteria & 2 & $\downarrow$ & $\downarrow$ & & & $\uparrow$ & & & $\downarrow$ & & & & \\
\hline \multicolumn{14}{|l|}{ Phylum Elusimicrobia } \\
\hline P_Elusimicrobia & 2 & & & & & $\leftrightarrow$ & & & & $\downarrow$ & & & \\
\hline G_Allobaculum & 10 & $\leftrightarrow, \downarrow(2), \uparrow(2)$ & $\uparrow$ & & & $\leftrightarrow, \uparrow$ & & $\uparrow(2)$ & & & & & \\
\hline \multicolumn{14}{|l|}{ Phylum Fusobacteria } \\
\hline P_Fusobacteria & 3 & $\downarrow, \uparrow$ & & & & $\leftrightarrow$ & & & & & & & \\
\hline \multicolumn{14}{|l|}{ Phylum Proteobacteria } \\
\hline P_Proteobacteria & 8 & $\downarrow(3), \uparrow$ & $\downarrow$ & & & $\leftrightarrow, \downarrow(2)$ & & & $\downarrow \uparrow$ & & & $\uparrow$ & \\
\hline C_Alphaproteobacteria & 2 & $\downarrow$ & & & & & & & & $\uparrow$ & & & \\
\hline G_Desulfovibrio & 5 & $\leftrightarrow, \downarrow$ & $\downarrow$ & & $\downarrow$ & $\uparrow$ & & & $\downarrow$ & & & & \\
\hline F_Desulfovibrionaceae & 4 & $\downarrow$ & $\downarrow$ & $\uparrow$ & & & & & $\downarrow$ & & & & \\
\hline F_Enterobacteriacae & 2 & $\uparrow$ & & & & & & & & & & $\downarrow$ & \\
\hline G_Escherichia & 3 & $\leftrightarrow, \uparrow$ & & & & & & & & & & & $\downarrow$ \\
\hline G_Helicobacter & 2 & $\downarrow$ & & & & $\downarrow$ & & & & & & & \\
\hline \multicolumn{14}{|l|}{ Phylum Tenericutes } \\
\hline P_Tenericutes & 6 & $\leftrightarrow, \uparrow$ & $\leftrightarrow$ & & & $\uparrow(2)$ & & & $\leftrightarrow, \uparrow$ & $\downarrow$ & & & \\
\hline C_Mollicutes & 2 & $\uparrow$ & & & & $\downarrow$ & & & & & & & \\
\hline \multicolumn{14}{|l|}{ Phylum Verrucomicrobia } \\
\hline P_Verrucomicrobia & 8 & $\leftrightarrow, \downarrow(2), \uparrow(5)$ & $\downarrow$ & & & $\uparrow$ & & & & & & & \\
\hline F_Verrucomicrobiaceae & 5 & $\uparrow(4)$ & $\downarrow(2)$ & & & & & & & & & & \\
\hline G_Akkermansia & 9 & $\uparrow(8)$ & & & & $\uparrow$ & & & & & & & \\
\hline S_Akkermansia muciniphila & 6 & $\leftrightarrow, \uparrow(3)$ & & & & $\uparrow(2)$ & & & & & & & \\
\hline
\end{tabular}

${ }^{a}$ Number of studies; M, metformin; A, acarbose; Mi, miglitol; Vo, voglibose; L, liraglutide; An, anagliptin; Sa, saxagliptin; Si, sitagliptin; Vi, vildagliptin; C, canagliflozin; D, dapagliflozin;

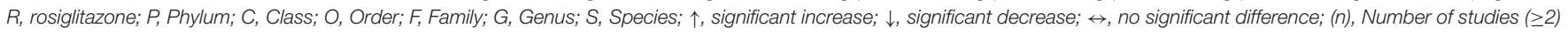
reported the same results.

secondary bile acids, while the other one (26) revealed an inverse trend. Concerning changes in total and primary bile acids, their levels in feces were unchanged among these three trials, but results in blood levels were conflicting $(21,26)$. The random trial assessing the effects of acarbose and glipizide on bile acid levels in newly diagnosed T2D patients (32) showed that acarbose might increase the plasma and fecal levels of primary bile acids, accompanied by decreases in secondary bile acids. In contrast, no significant changes in bile acid levels were found in patients treated with glipizide. 
TABLE 7 | Consistent and inconsistent effects of each anti-hyperglycemic drug on specific taxa in mouse gut microbiota, categorized by mice or rat models with three distinct animal models.

\begin{tabular}{|c|c|c|c|c|c|c|c|c|c|}
\hline \multirow[t]{2}{*}{ Specific taxa } & \multirow[t]{2}{*}{ Phylum } & \multirow[t]{2}{*}{$N^{a}$} & \multirow[t]{2}{*}{ Trend $^{b}$} & \multicolumn{3}{|c|}{ Mice models } & \multicolumn{3}{|c|}{ Rat models } \\
\hline & & & & Normal & $\begin{array}{c}\text { Dietary or } \\
\text { STZ }\end{array}$ & $\begin{array}{c}\text { Gene } \\
\text { knockout }\end{array}$ & Normal & $\begin{array}{c}\text { Dietary or } \\
\text { STZ }\end{array}$ & $\begin{array}{c}\text { Gene } \\
\text { knockout }\end{array}$ \\
\hline \multicolumn{10}{|l|}{ METFORMIN } \\
\hline \multicolumn{10}{|l|}{ Consistent results } \\
\hline Firmicutes/Bacteroidetes & & 2 & $\downarrow$ & & $\downarrow(2)$ & & & & \\
\hline F_Porphyromonadaceae & Bacteroidetes & 2 & $\uparrow$ & $\uparrow$ & $\uparrow$ & & & & \\
\hline F_Prevotellaceae & Bacteroidetes & 2 & $\uparrow$ & $\uparrow$ & & & & $\uparrow$ & \\
\hline F_Rikenellaceae & Bacteroidetes & 2 & $\uparrow$ & $\uparrow(2)$ & & & & & \\
\hline G_Butyricimonas & Bacteroidetes & 2 & $\uparrow$ & & $\uparrow$ & $\uparrow$ & & & \\
\hline F_Enterococcaceae & Firmicutes & 2 & $\downarrow$ & & $\downarrow$ & & & $\downarrow$ & \\
\hline F_Streptococcaceae & Firmicutes & 2 & $\downarrow$ & & $\downarrow$ & & & $\downarrow$ & \\
\hline F_Verrucomicrobiaceae & Verrucomicrobia & 4 & $\uparrow$ & $\uparrow(2)$ & $\uparrow(3)$ & & & & \\
\hline G_Akkermansia & Verrucomicrobia & 8 & $\uparrow$ & & $\uparrow(6)$ & $\uparrow$ & & $\uparrow$ & \\
\hline S_Akkermansia spp. & Verrucomicrobia & 2 & $\uparrow$ & $\uparrow$ & & & & & $\uparrow$ \\
\hline F_Alcaligenaceae & Proteobacteria & 2 & $\uparrow$ & & $\uparrow$ & & & $\uparrow$ & \\
\hline \multicolumn{10}{|l|}{ Inconsistent results } \\
\hline P_Bacteroidetes & Bacteroidetes & 8 & $\uparrow$ & $\leftrightarrow$ & $\downarrow, \uparrow(4)$ & & & $\leftrightarrow, \uparrow$ & $\leftrightarrow$ \\
\hline G_Bacteroides & Bacteroidetes & 7 & $\uparrow$ & $\uparrow$ & $\leftrightarrow, \downarrow, \uparrow(2)$ & $\leftrightarrow$ & & $\uparrow(2)$ & \\
\hline G_Odoribacter & Bacteroidetes & 3 & $\downarrow$ & & $\downarrow$ & $\leftrightarrow$ & & & $\downarrow$ \\
\hline G_Parabacteroides & Bacteroidetes & 5 & $\uparrow$ & $\downarrow$ & $\downarrow, \uparrow(4)$ & & & & \\
\hline G_Prevotella & Bacteroidetes & 4 & $\uparrow$ & & $\uparrow$ & $\downarrow$ & & $\uparrow(2)$ & \\
\hline P_Firmicutes & Firmicutes & 8 & $\circ$ & $\leftrightarrow$ & $\leftrightarrow, \uparrow, \downarrow(3)$ & & & $\leftrightarrow(2)$ & $\uparrow$ \\
\hline G_Allobaculum & Firmicutes & 5 & $\circ$ & $\downarrow$ & $\uparrow, \downarrow$ & $\leftrightarrow$ & & $\uparrow$ & \\
\hline G_Anaerotruncus & Firmicutes & 2 & $\circ$ & & $\downarrow$ & $\leftrightarrow$ & & & \\
\hline G_Blautia & Firmicutes & 2 & $\circ$ & & $\downarrow$ & & & $\uparrow$ & \\
\hline G_Christensenella & Firmicutes & 2 & $\circ$ & & $\uparrow$ & $\leftrightarrow$ & & & \\
\hline G_Coprococcus & Firmicutes & 3 & $\downarrow$ & & $\downarrow(2)$ & $\uparrow$ & & & \\
\hline F_Dehalobacteriaceae & Firmicutes & 2 & $\circ$ & & $\downarrow$ & & & & $\uparrow$ \\
\hline G_Dehalobacterium & Firmicutes & 2 & $\circ$ & & $\downarrow$ & & & & $\uparrow$ \\
\hline G_Enterococcus & Firmicutes & 5 & $\downarrow$ & & $\downarrow(2)$ & & & $\leftrightarrow, \uparrow, \downarrow$ & \\
\hline F_Lactobacillaceae & Firmicutes & 2 & $\circ$ & & & & & $\uparrow, \downarrow$ & \\
\hline G_Lactobacillus & Firmicutes & 8 & $\uparrow$ & & $\uparrow, \downarrow$ & $\uparrow$ & & $\downarrow, \uparrow(3)$ & $\uparrow$ \\
\hline G_Lactococcus & Firmicutes & 2 & $\circ$ & & $\uparrow, \downarrow$ & & & & \\
\hline G_Oscillospira & Firmicutes & 2 & $\circ$ & & $\uparrow, \downarrow$ & & & & \\
\hline G_Roseburia & Firmicutes & 2 & $\circ$ & & & $\leftrightarrow$ & & $\downarrow$ & \\
\hline G_Ruminococcus & Firmicutes & 4 & $\circ$ & $\uparrow$ & $\downarrow(2)$ & $\uparrow$ & & & \\
\hline G_Turicibacter & Firmicutes & 3 & $\circ$ & $\uparrow$ & & $\leftrightarrow, \downarrow$ & & & \\
\hline G_Bifidobacterium & Actinobacteria & 4 & $\circ$ & & $\leftrightarrow$ & $\leftrightarrow$ & & $\uparrow, \downarrow$ & \\
\hline P_Fusobacteria & Fusobacteria & 2 & $\circ$ & & $\uparrow$ & & & & $\downarrow$ \\
\hline C_Fusobacteriia & Fusobacteria & 2 & $\circ$ & & $\uparrow$ & & & & $\downarrow$ \\
\hline O_Fusobacteriales & Fusobacteria & 2 & $\circ$ & & $\uparrow$ & & & & $\downarrow$ \\
\hline P_Proteobacteria & Proteobacteria & 4 & $\downarrow$ & & $\downarrow$ & & & $\uparrow, \downarrow$ & $\downarrow$ \\
\hline G_Desulfovibrio & Proteobacteria & 2 & $\circ$ & & $\downarrow$ & $\leftrightarrow$ & & & \\
\hline G_Escherichia & Proteobacteria & 2 & $\circ$ & & $\uparrow$ & & & $\leftrightarrow$ & \\
\hline S_Escherichia coli & Proteobacteria & 2 & $\circ$ & & $\downarrow$ & & & $\leftrightarrow$ & \\
\hline G_Klebsiella & Proteobacteria & 2 & $\circ$ & & & & & $\leftrightarrow, \uparrow$ & \\
\hline
\end{tabular}


TABLE 7 | Continued

\begin{tabular}{|c|c|c|c|c|c|c|c|c|c|}
\hline Specific taxa & Phylum & $N^{a}$ & Trend $^{\mathrm{b}}$ & \multicolumn{3}{|c|}{ Mice models } & \multicolumn{3}{|c|}{ Rat models } \\
\hline G_Parasutterella & Proteobacteria & 3 & $\uparrow$ & $\uparrow$ & $\leftrightarrow$ & & & $\uparrow$ & \\
\hline G_Proteus & Proteobacteria & 2 & $\circ$ & & $\uparrow$ & $\downarrow$ & & & \\
\hline G_Sutterella & Proteobacteria & 3 & $\uparrow$ & & $\uparrow$ & $\leftrightarrow$ & & $\uparrow$ & \\
\hline P_Tenericutes & Tenericutes & 2 & $\circ$ & & $\uparrow$ & & & & $\leftrightarrow$ \\
\hline P_Verrucomicrobia & Verrucomicrobia & 7 & $\uparrow$ & $\leftrightarrow$ & $\uparrow(4)$ & & & $\uparrow, \downarrow$ & $\downarrow$ \\
\hline S_Akkermansia muciniphila & Verrucomicrobia & 4 & $\uparrow$ & & $\leftrightarrow, \uparrow(3)$ & & & & \\
\hline G_AF12 & NA & 2 & 。 & & $\downarrow$ & $\leftrightarrow$ & & & \\
\hline
\end{tabular}

\section{ACARBOSE}

\section{Consistent result}

F_Lactobacillaceae

\section{Inconsistent results}

\section{F_S24-7}

P_Firmicutes

F_Erysipelotrichaceae

G_Lachnospiraceae UCG-001

G_Lactobacillus

G_Ruminococcus 2

\section{DAPAGLIFLOZIN}

Inconsistent result

G_Enterococcus

\section{LIRAGLUTIDE}

\section{Consistent results}

Firmicutes/Bacteroidetes

G_Blautia

S_Akkermansia muciniphila

Inconsistent results

P_Actinobacteria

P_Bacteroidetes

G Bacteroides

C_Bacteroidia

O_Bacteroidales

F_Porphyromonadaceae

G_Prevotella_9

P_Firmicutes

G_Allobaculum

G_Christensenellaceae_R_7_group

F_Lachnospiraceae

F_Ruminococcaceae

G_Lactobacillus

G_Roseburia

P Proteobacteria

P Tenericutes

P_Verrucomiacrobia

\section{Firmicutes}

Bacteroidetes

Firmicutes

Firmicutes

Firmicutes

Firmicutes

Firmicutes

2

$\downarrow$

$\downarrow$

$\leftrightarrow$

$\leftrightarrow$

$\uparrow$

2

$\uparrow, \downarrow$

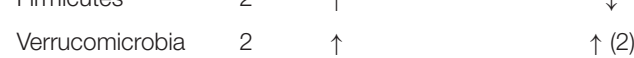

Actinobacteria $2 \quad \circ$

Bacteroidetes $3 \uparrow \uparrow$

Bacteroidetes 3

Bacteroidetes

Bacteroidetes

Bacteroidetes

Bacteroidetes

Firmicutes

Firmicutes

Firmicutes

Firmicutes

Firmicutes

Firmicutes

Firmicutes

Proteobacteria

Tenericutes

Verrucomiacrobia
2

2

2

2

2

3

2

2

2

2

2

2

2
3

2


TABLE 7 | Continued

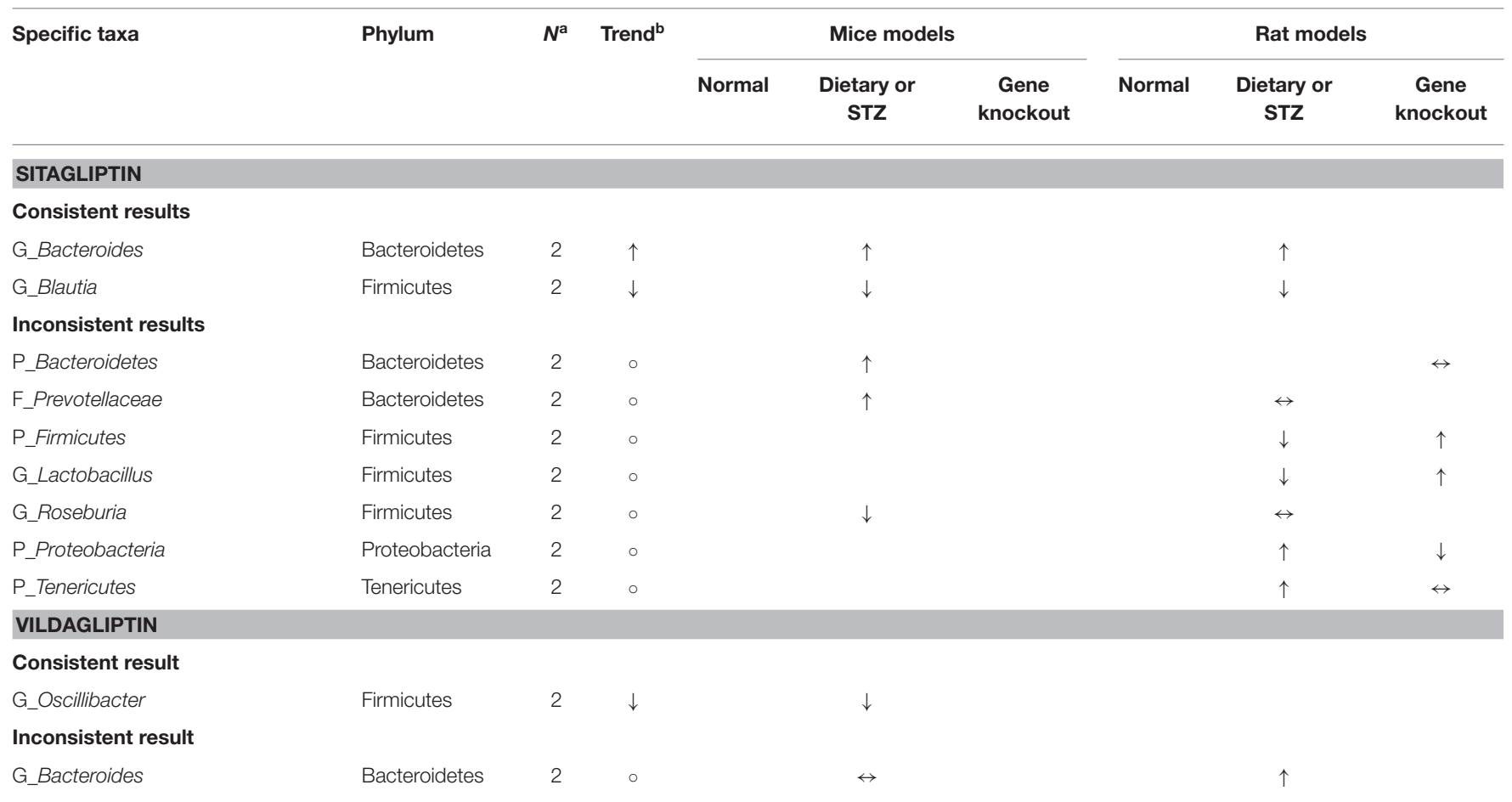

P, Phylum; C, Class; O, Order; F, Family; G, Genus; ${ }^{a}$ Number of studies; ${ }^{b}$ Trend of alteration (reported in > 50\% of studies): $\uparrow$, a trend of increase; $\downarrow$, a trend of decrease; o, inconclusive results; Alteration of specific taxa: $\uparrow$, significant increase; $\downarrow$, significant decrease; $\leftrightarrow$, no significant difference; (n), number of papers $(\geq 2)$ reported the same results.

TABLE 8 | Effects of anti-hyperglycemic drug on diversity of human gut microbiota.

\begin{tabular}{|c|c|c|c|c|c|}
\hline Drugs & Object & \multicolumn{2}{|c|}{$\alpha$-diversity } & $\beta$-diversity ${ }^{c}$ & References \\
\hline & & ns & ns & $\neq$ & (22) \\
\hline & Obese & ns & ns & ns & (19) \\
\hline & Newly T2D & ns & $\uparrow$ & $\neq$ & (20) \\
\hline & Prevalent T2D & - & - & $\neq$ & (21) \\
\hline & & ns & ns & - & (23) \\
\hline & & - & $\downarrow$ & $\neq$ & (27) \\
\hline & & - & ns & ns & (28) \\
\hline & & ns & - & ns & (29) \\
\hline Liraglutide & Prevalent T2D & - & - & $\neq$ & (34) \\
\hline Glipizide & Newly T2D & ns & ns & - & (32) \\
\hline
\end{tabular}

${ }^{a}$ Richness was assessed by Chao 1, ACE, and Rarefaction indices, gene count, number of OTUs, or number of species; ${ }^{b}$ Evenness was assessed by Shannon, Simpson indices; ${ }^{c} \beta$ diversity was assessed by UniFrac (weighted, unweighted), Bray-Curtis, Jensen-Shannon, or Jaccard distances using Principal Component Analysis (PCA) and Principal Coordinates Analysis (PCOA); $\uparrow$, significant increase; $\downarrow$, significant decrease; $\neq$, significant difference; ns, no significant difference; -, no information. 
TABLE 9 | Effects of anti-hyperglycemia drugs on diversity in mouse gut microbiota.

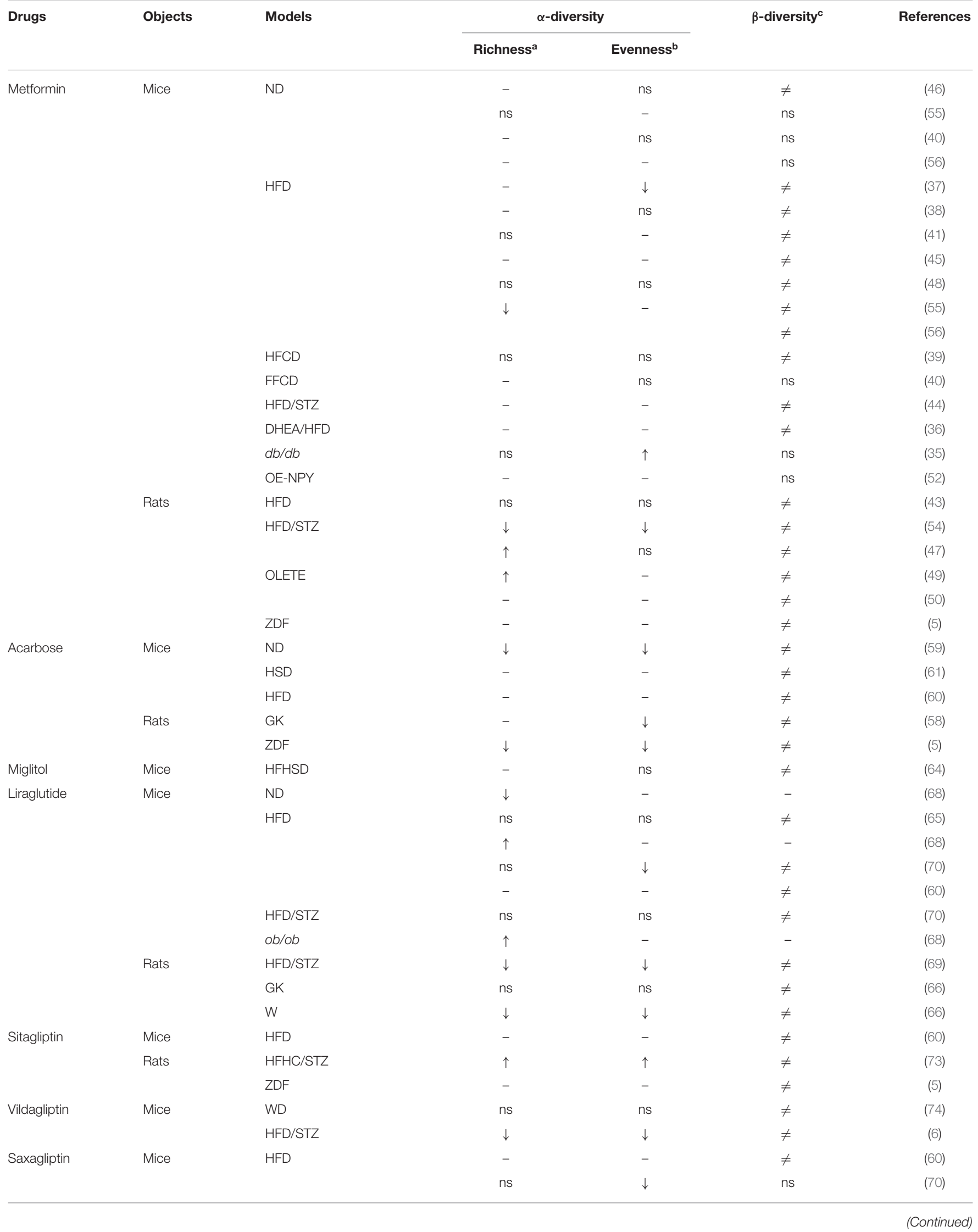


TABLE 9 | Continued

\begin{tabular}{|c|c|c|c|c|c|c|}
\hline Drugs & Objects & Models & \multicolumn{2}{|c|}{$\alpha$-diversity } & $\beta$-diversity ${ }^{c}$ & References \\
\hline Anagliptin & Rats & OLETF \& PS & ns & ns & - & (71) \\
\hline Dapagliflozin & Mice & MafA-deficient & $\downarrow$ & $\downarrow$ & ns & (75) \\
\hline Pioglitazone & Mice & KKAy & - & $\downarrow$ & $\neq$ & (78) \\
\hline
\end{tabular}

${ }^{a}$ Richness was assessed by Chao1, ACE, and Rarefaction indices, gene count, number of OTUs, or number of species; ${ }^{b}$ Evenness was assessed by Shannon, Simpson indices; ${ }^{c} \beta$ -

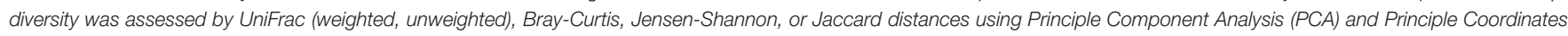
Analysis (PCOA); $\uparrow$, significant increase; $\downarrow$, significant decrease; $\neq$, significant difference; ns, no significant difference; -, no information.

As for rodent studies, one study in rats (51) revealed that the fecal level of total bile acids increased while the levels in liver tissues were decreased after metformin intervention. One study in mice (63) found that voglibose treatment was associated with increases in serum levels of primary bile acids, accompanied by decreases in serum levels of secondary bile acids (Supplementary Table 4).

\section{Associations With Host Metabolic Parameters}

Among pre-diabetic and T2D patients treated with metformin $(20,21)$ or acarbose $(7,32,33)$, alterations in certain specific taxa in human gut microbiota were associated with improvement in $\mathrm{HbA1C}$ and fasting blood glucose values, body weights, and lipid profiles (Table 10). For instance, increments in the genera Escherichia, Shigella, Subdoligranulum, and Dialister, and the species Bifidobacterium adolescentis, Bifidobacterium longum, and Lactobacillus gasseri were inversely associated with $\mathrm{HbA1C}$ after treating with metformin or acarbose $(7,20,21,32)$. In addition, there were inverse associations between increases in the genus Blautia and fasting blood glucose after treating with metformin (20).

Mouse studies treated with metformin (44, 50, 55, 57), liraglutide $(65,66,70)$, and saxagliptin (70) also explored the relationship between changes in the compositions of gut microbiota and improvement in various metabolic parameters (Table 11). It was found that some related specific taxa after treating with metformin in mice (i.e., Bacteroides spp., Blautia) were different from that in humans.

\section{DISCUSSION}

Our study provides a comprehensive review to report human and animal data separately about reciprocal interactions between anti-hyperglycemic drugs and specific taxonomic groups of gut microbiota. While other reviews suggest the effects of antihyperglycemic drugs on gut microbiota without discerning findings from either human or animal studies $(8,11,13)$, this systematic review attempts to fill the gap of these reviews to try to explore the associations among anti-hyperglycemic agents, specific taxonomic patterns of gut microbiota, and glucose control or metabolic profiles mainly in humans, as compared to those reported in mouse studies. Further, the fact that threequarters of included studies were published in and after 2017 implies a growing interest in this clinical question for an up-todate systematic review.

Of the 17 human studies selected, the majority of these studies focus on either newly diagnosed or prevalent T2D patients, and directed toward investigating the interplay of metformin, and to a lesser extent, acarbose, with gut microbiota. Our results suggest that these two drugs mediate their glucose-lowering effect, in part, by stimulating beneficial gut bacteria that could produce metabolites to promote intestinal homeostasis $(3,9)$. We rationalize that alterations in gut microbiota compositions might also underlie the gastrointestinal side effects known to metformin, i.e., diarrhea and fecal incontinence $(10,25,80)$. In contrast, results from other anti-hyperglycemic drugs analyzed in this study showed inconsistency with respect to their effects on the compositions of gut microbiota, which might be attributable to small numbers of studies and, equally important, differences in animal models and experimental conditions used among these studies.

Further, treatment durations of anti-hyperglycemic drugs in available studies, regardless of human or animal, varied to a great extent (i.e., few days to few months). Thus, the reported drug effects on the gut microbiota structure were diverse. Indeed, the anti-hyperglycemic drugs, i.e., metformin, could affect the intestinal bacterial compositions after 1 or several days of treatment $(24,25,40,43)$, or after prolonged periods of treatments (21). For instance, $\mathrm{Wu}$ et al. found that gut microbiota compositions after a 2- and 4-month treatment of metformin in newly T2D patients were not identical (21). In contrast, Wang et al. did not find significant changes in the gut microbiota compositions among T2D patients after different periods of metformin or liraglutide treatment, given their baseline gut microbiota compositions were unknown (34). Thus, there are no consistent findings on gut microbiota after various treatment durations of anti-hyperglycemic drugs, and further studies are warranted to explore the treatment duration of anti-hyperglycemics required for emergence of beneficial gut bacteria. 
TABLE 10 | Association between specific taxa and human metabolic parameters.

\begin{tabular}{|c|c|c|c|c|c|c|}
\hline Parameters & Association & Specific taxa & Alteration & Drugs & Participants & References \\
\hline \multirow[t]{4}{*}{$\mathrm{HbA} 1 \mathrm{C}$} & Negative & $\begin{array}{l}\text { G_Escherichia } \\
\text { G_Shigella }\end{array}$ & $\uparrow$ & $\mathrm{M}$ & Newly T2D & (20) \\
\hline & & $\begin{array}{l}\text { S_Bifidobacterium } \\
\text { adolescentis }\end{array}$ & $\uparrow$ & $\mathrm{M}$ & Newly T2D & $(21)$ \\
\hline & & $\begin{array}{l}\text { S_Lactobacillus gasseri } \\
\text { S_Bifidobacterium longum }\end{array}$ & $\uparrow$ & A & Newly T2D & (32) \\
\hline & & $\begin{array}{l}\text { G_Subdoligranulum } \\
\text { G_Dialister }\end{array}$ & $\uparrow$ & A & Pre-diabetic & $(7)$ \\
\hline Fasting blood glucose & Negative & G_Blautia & $\uparrow$ & M & Newly T2D & (20) \\
\hline \multirow[t]{2}{*}{ Body weight } & Positive & $\begin{array}{l}\text { S_Bacteroides plebeius } \\
\text { S_Bacteroides dorei } \\
\text { S_Bacteroides vulgatus } \\
\text { S_Clostridium bolteae }\end{array}$ & $\downarrow$ & A & Newly T2D & $(32)$ \\
\hline & Negative & $\begin{array}{l}\text { S_Lactobacillus gasseri } \\
\text { S_Bifidobacterium longum }\end{array}$ & $\uparrow$ & A & Newly T2D & $(32)$ \\
\hline HDL cholesterol & Positive & S_Bidifobacterium longum & $\uparrow$ & A & Prevalent T2D & (33) \\
\hline LDL cholesterol & Negative & G_Blautia & $\uparrow$ & $\mathrm{M}$ & Newly T2D & (20) \\
\hline
\end{tabular}

G, genus; S, species; M, metformin; A, acarbose; $\uparrow$, significant increase; $\downarrow$, significant decrease.

Evidence indicates that the use of metformin or acarbose in T2D patients was associated with increases in the abundance of beneficial bacteria, including the genera Bifidobacterium (phylum Actinobacteria) and Lactobacillus (phylum Firmicutes), and the species A. muciniphila (phylum Verrucomicrobia). The increase in the genus Bifidobacterium was positively associated with diabetes control, which is consistent with that reported in the review by Gurung et al. (1). In addition, two included studies showed an increase in two specific species of the genus Bifidobacterium (B. adolescentis and B. longum), which was inversely associated with $\mathrm{HbA1C}$ levels or body weights, and positively associated with HDL cholesterol levels among newly diagnosed T2D patients $(21,32)$.

A number of human studies have reported positive associations between the abundance of the genus Lactobacillus (phylum Firmicutes) and improved T2D control (81, 82). For example, T2D patients treated with acarbose showed increased L. gasseri levels, accompanied by lower HbA1C and body weights (32). In addition, as several species in the genus Lactobacillus have been used as probiotics, administration of these Lactobacillus strains showed beneficial effects on glycemic control and lipid profiles in T2D patients (4). Moreover, almost all animal studies that tested the efficacy of several species from the genus as probiotics for T2D reported improvements of glucose parameters (1).

A previous report found decreased abundance in the mucindegrading bacterium A. municiphila in patients with metabolic disorders, including obesity, impaired glucose tolerance, and diabetes, which were associated with insulin resistance, dyslipidemia, and overweight (83). Two other studies showed increased amounts of $A$. municiphila in newly diagnosed and prevalent T2D patients treated with metformin, which, however, did not provide pertinent information on metabolic parameters. The potential role of this mucin-degrading bacterium in ameliorating metabolic disorders was further confirmed by a series of animal experiments. For example, mice treated with metformin and liraglutide showed increased levels of $A$. municiphila in association with improved control of blood glucose and body weight $(55,65)$. More importantly, HFD-fed mice treated with $A$. municiphila exhibited similar improvements in glucose tolerance and goblet cell production and inflammatory regulations as compared to the metformin treatment group (56).

The effect of metformin and acarbose on the abundance of different species of the genus Bacteroides (phylum Bacteroidetes) is interesting. The genus Bacteroides seem to play a beneficial role in glucose metabolism where B. intestinalis and B. vulgatus were decreased in T2D patients, and B. stercoris was enriched in patients with diabetes remission (1). The same phenomenon was also noted in experimental animals (1). However, decreased abundance of some Bacteroides species, including B. pleibeius, B. dorei, B. vulgatus, after using acarbose in newly diagnosed T2D patients was reported to be positively associated with body weight in one study (32). As for rodent studies, colonization of $B$. fragilis was associated with more severe glucose intolerance in HFD-fed mice (24). A recent study, which compared fecal microbiota compositions between T2D patients and non-diabetic individuals, showed that Bacteroides was an independent risk factor of the disease by diminishing insulin sensitivity (84).

The effects of metformin on A. muciniphila were similar in both human and rodent studies. However, there was an inverse association of Bacteroides and metformin use in human and mouse studies in this review. Alterations of many other taxa in humans treated with metformin or acarbose were not the same as in mouse studies, and vice versa. The diverse dietary habits, metabolism or inflammatory statuses of host, body sizes and organs in these human and mouse studies might contribute to inconsistent findings of gut microbiota compositions $(12,85)$. Although the gut microbiota of human 
TABLE 11 | Association between specific taxa and mouse metabolic parameters.

\begin{tabular}{|c|c|c|c|c|c|c|}
\hline Parameters & Association & Specific taxa & Alteration & Drugs & Models & References \\
\hline \multirow[t]{3}{*}{ Fasting blood glucose } & Positive & S_Bacteroides spp. & $\downarrow$ & $\mathrm{M}$ & HFD/STZ mice & $(44)$ \\
\hline & Negative & S_Akkermansia muciniphila & $\uparrow$ & $\mathrm{M}$ & HFD mice & $(55)$ \\
\hline & & S_Bifidobacterium spp. & $\downarrow$ & $M$ & HFHSD rats & $(57)$ \\
\hline \multirow[t]{6}{*}{ Body weight } & Positive & $\begin{array}{l}\text { G_Candidatus Arthromitus } \\
\text { G_Roseburia } \\
\text { G_Marvinbryantia }\end{array}$ & $\downarrow$ & $\mathrm{L}$ & HFD/STZ mice & $(70)$ \\
\hline & & $\begin{array}{l}\text { S_Clostridia sp., } \\
\text { S_Clostridiales spp. } \\
\text { S_Oscillospiraceae sp. } \\
\text { S_Erysipelatoclostridium sp. }\end{array}$ & $\downarrow$ & $L$ & HFD mice & (65) \\
\hline & & $\begin{array}{l}\text { S_Anaerotruncus sp. } \\
\text { G3(2012) } \\
\text { S_Firmicutes sp. } \\
\text { S_Bacteroidales sp. }\end{array}$ & & & & \\
\hline & Negative & $\begin{array}{l}\text { S_Clostridiales spp. } \\
\text { S_Oscillospiraceae sp. } \\
\text { S_Burkholderiales } \\
\text { bacterium YL45 } \\
\text { S_Akkermansia muciniphila }\end{array}$ & $\uparrow$ & $L$ & HFD mice & $(65)$ \\
\hline & & $\begin{array}{l}\text { G_Lactobacillus } \\
\text { G_Turicibacter } \\
\text { G_Anaerostipes } \\
\text { G_Allobaculum } \\
\text { G_Blautia }\end{array}$ & $\uparrow$ & $L$ & HFD/STZ mice & $(70)$ \\
\hline & & $\begin{array}{l}\text { G_Lactobacillus } \\
\text { G_Turicibacter } \\
\text { G_Allobaculum }\end{array}$ & $\uparrow$ & $\mathrm{Sa}$ & HFD/STZ mice & $(70)$ \\
\hline \multirow[t]{2}{*}{ LDL cholesterol } & Positive & G_Romboutsia & $\downarrow$ & $\mathrm{L}$ & HFD/GK rats & $(66)$ \\
\hline & Negative & G_Prevotella & $\uparrow$ & $\mathrm{L}$ & HFD/GK rats & $(66)$ \\
\hline \multirow[t]{6}{*}{ Total cholesterol } & Positive & S_Prevotella spp. & $\downarrow$ & M & OLETF rats & $(50)$ \\
\hline & & $\begin{array}{l}\text { S_Clostridia sp. } \\
\text { S_Clostridiales spp. } \\
\text { S_Oscillospiraceae sp. } \\
\text { S_Erysipelatoclostridium sp. }\end{array}$ & $\downarrow$ & $L$ & HFD mice & $(65)$ \\
\hline & & $\begin{array}{l}\text { S_Anaerotruncus sp. } \\
\text { G3(2012) } \\
\text { S_Firmicutes sp. } \\
\text { S_Bacteroidales sp. }\end{array}$ & & & & \\
\hline & & G_Romboutsia & $\downarrow$ & $\mathrm{L}$ & HFD/GK rats & $(66)$ \\
\hline & & S_Clostridium cocleatum & $\uparrow$ & M & HFD mice & (55) \\
\hline & Negative & G_Prevotella & $\uparrow$ & $\mathrm{L}$ & HFD/GK rats & $(66)$ \\
\hline \multirow[t]{3}{*}{ Triglyceride } & Positive & S_Prevotella spp. & $\downarrow$ & $\mathrm{M}$ & OLETF rats & $(50)$ \\
\hline & & G_Romboutsia & $\downarrow$ & $L$ & HFD/GK rats & $(66)$ \\
\hline & Negative & G_Prevotella & $\uparrow$ & $L$ & HFD/GK rats & $(66)$ \\
\hline
\end{tabular}

G, Genus; S, Species; M, metformin; Sa, saxagliptin; L, liraglutide; $\uparrow$, significantly increase; $\downarrow$, significantly decrease.

and mice are dominated by two major phyla, i.e., Bacteroidetes and Firmicutes, approximately $85 \%$ of the representative gut microbiota sequences in mice were not found in humans (86).

Further, the genera Escherichia and Shigella, belonging to the order of Enterobacterales in phylum Proteobacteria, were found to increase consistently after metformin treatment in T2D patients. Certain bacteria belonging to the phylum Proteobacteria, including the order Enterobacteriales, was found to be overly present in patients with metabolic disorders and $\mathrm{T} 2 \mathrm{D}$, and were positively related to intestinal permeability and endotoxemia in the pathophysiology of these metabolic diseases $(87,88)$. Enrichment in the order Enterobacterales, especially Escherichia coli, was demonstrated to play an important role in gut inflammation in patients with inflammatory bowel disease and also in various mouse model of colitis (88). Elbere et al. (25) observed an association between the severity of gastrointestinal 
side effects and increased abundance of the genera Escherichia and Shigella. Thus, the enrichment of the order Enterobacteriales might contribute to gastrointestinal side effects of metformin.

In this review, the results of $\beta$-diversity indicate significant changes in gut microbiome structure related to metformin or acarbose treatment. The findings on $\alpha$-diversity among those treated with metformin were inconsistent while the richness and evenness were decreased after treating with acarbose. For healthy human subjects, the reference microbiome list and abundance profile showed various ratios of Bacteroidetes and Firmicutes, as well as the other phyla, e.g., Acinobacteria, Proteobacteria (89). This might reflect sufficient $\alpha$ - and $\beta$-diversities in healthy individuals due to significant regional heterogeneity at the species level and consistency at the higher taxonomic level (89). With regard to T2D patients, the associations between the disease and the diversities of microbiota were inconclusive (1). In the other words, there is no consensus or simple way to make a conclusion on the relationship between diversity and gut microbiota compositions among T2D patients treated with metformin or acarbose.

While metformin and acarbose have been shown to stimulate the growth of SCFA-producing bacteria, e.g., Lactobacillus and Bifidobacterium, information on the effects of these drugs on the fecal levels of various SCFAs in humans is lacking in the literature. Thus, this review entailed data from mouse studies, which are more informative. These mouse studies showed an increase trend in fecal and cecal levels of acetate, propionate, and butyrate in response to treatments of metformin, acarbose, voglibose, dapagliflozin or canagliflozin, of which the impacts on other physiological functions, other than that in gastrointestinal track, warrant further evaluations (90). With respect to bile acids, information on the effects of anti-hyperglycemic agents on their levels is limited and often conflicting in human vs. rodent studies, which merits further investigations to understand the role of other confounding factors, such as diets, antibiotic therapy, and disease states (91).

Again, the most critical limitation is lack of consistency among human and rodent studies. In humans, differences in the health status of participants, disease type or staging, ethnicity, drug dosage, and duration of treatment might directly impact gut microbiota compositions. Furthermore, it becomes difficult to come to a conclusion due to the small number of participants as well as differences in study design in each study. The risk of bias of studies needs to be taken into considerations, in which overall bias of randomized trials was high risk and unclear risk, and two out of five quasi-experimental studies and all of observational studies were at serious risk. Also, there was a high degree of heterogeneity in rodent studies due to differences in species used and environmental factors, as alluded above. Differences in microbiota analysis methods could also be a cause of deviation.

Another major limitation is lack of human studies on antihyperglycemic drugs beyond metformin and acarbose. Thus, no conclusion could be reached regarding the associations between human gut microbiota and these drugs. Because results from rodent models might not always be translatable to humans, conclusions should be made with cautions.
Although findings from rodent studies included in this review suggest potential positive effects of other antihyperglycemic drugs besides metformin and acarbose on human gut microbiota, additional human studies on these drugs are needed to clarify the role of gut microbiota in their therapeutic efficacies.

In light of the enormous amounts of published data, this systematic review aims to provide readers a comprehensive view of this emerging area by taking an integrated approach through an all-inclusive literature search in conjunction with vigorous data extraction and validation, and assessment of risk bias. Moreover, this systematic review has tried to differentiate various aspects of the anti-hyperglycemic drug-gut microbiomehost axis, thereby filling the gap of merging all available data from human or animal studies relevant to the interdependence between anti-hyperglycemic drugs and the specific taxon of gut microbiota. Nevertheless, more investigations are warranted to support the positive contribution of metformin and acarbose to the health of gut microbiome (e.g., A. muciniphila, Lactobacillus, Bifidobacterium longum). In addition, given the limited information available in the literature, more studies are needed to shed light onto the roles of other anti-hyperglycemic drugs (e.g., miglitol, voglibose) in modulating human host taxa of gut microbiome.

\section{CONCLUSION}

This review highlights that changes in specific taxa and $\beta$ diversity of gut microbiota were associated with metformin and acarbose in humans while pertinent information for other antihyperglycemic drugs could be only obtained in rodent studies. These results support the possible action mechanisms of these drugs, which may have translational potential to foster new approaches for the treatment of diseases related to gut dysbiosis in the future. Mouse studies on the other anti-hyperglycemics suggested the links between these drugs and gut microbiota were inconclusive. Therefore, additional human studies are needed to explore the role of gut microbiota in their therapeutic efficacies or side effects.

\section{DATA AVAILABILITY STATEMENT}

The original data presented in the study are included in the article/Supplementary Material, further inquiries can be directed to the corresponding author.

\section{AUTHOR CONTRIBUTIONS}

TC, P-CL, H-WL, CC, C-SC, K-CW, J-LH, and L-YY collected, screened, and extracted the data and analyzed the results. TC, H-WL, CC, and L-YY wrote the first draft of the manuscript. All authors contributed to conception, design of the study, manuscript revision, read, and approved the submitted version. 


\section{FUNDING}

This research was partially supported by China Medical University under Grant CMU107-Z-03, CMU108-N-03, CMU107-Z-03, CMU-108-Z-07, and 1075955A; Ministry of Science and Technology under Grant MOST 109-2320-B039-023, 109-2622-8-039-002-TB1, and 108-2911-I-039-301; Ministry of Education Center-of-Excellence under Grant for Drug Development Center, China Medical University, Taichung, Taiwan. The funding agencies played no role in the study implementation, analysis or interpretation of data, or preparation and review or approval of the manuscript.

\section{ACKNOWLEDGMENTS}

We would like to appreciate Shu-Han Yang, Fang-Chu Lin, YuChing Wang, Yu-Tong Wang, Shang-Hua Wu, Po-Chen Chu, and those who were not listed but helped to search and review the literature in this project.

\section{REFERENCES}

1. Gurung M, Li Z, You H, Rodrigues R, Jump DB, Morgun A, et al. Role of gut microbiota in type 2 diabetes pathophysiology. EBioMedicine. (2020) 51:102590. doi: 10.1016/j.ebiom.2019.11.051

2. Qin J, Li Y, Cai Z, Li S, Zhu J, Zhang F, et al. A metagenome-wide association study of gut microbiota in type 2 diabetes. Nature. (2012) 490:5560. doi: 10.1038/nature11450

3. Adeshirlarijaney A, Gewirtz AT. Considering gut microbiota in treatment of type 2 diabetes mellitus. Gut microbes. (2020) 11:253-64. doi: 10.1080/19490976.2020.1717719

4. Tiderencel KA, Hutcheon DA, Ziegler J. Probiotics for the treatment of type 2 diabetes: a review of randomized controlled trials. Diabetes Metab Res Rev. (2020) 36:e3213. doi: 10.1002/dmrr.3213

5. Zhang M, Feng R, Yang M, Qian C, Wang Z, Liu W, et al. Effects of metformin, acarbose, and sitagliptin monotherapy on gut microbiota in zucker diabetic fatty rats. BMJ Open Diabetes Res Care. (2019) 7:e000717. doi: 10.1136/bmjdrc-2019-000717

6. Zhang Q, Xiao X, Li M, Yu M, Ping F, Zheng J, et al. Vildagliptin increases butyrate-producing bacteria in the gut of diabetic rats. PLoS ONE. (2017) 12:e0184735. doi: 10.1371/journal.pone.0184735

7. Zhang X, Fang Z, Zhang C, Xia H, Jie Z, Han X, et al. Effects of acarbose on the gut microbiota of prediabetic patients: a randomized, double-blind, controlled crossover trial. Diabetes Ther. (2017) 8:293307. doi: 10.1007/s13300-017-0226-y

8. Whang A, Nagpal R, Yadav H. Bi-directional drug-microbiome interactions of anti-diabetics. EBioMedicine. (2019) 39:591602. doi: 10.1016/j.ebiom.2018.11.046

9. Kyriachenko Y, Falalyeyeva T, Korotkyi O, Molochek N, Kobyliak N. Crosstalk between gut microbiota and antidiabetic drug action. World J Diabetes. (2019) 10:154-68. doi: 10.4239/wjd.v10.i3.154

10. Stein SA, Lamos EM, Davis SN. A review of the efficacy and safety of oral antidiabetic drugs. Expert Opin Drug Saf. (2013) 12:153-75. doi: 10.1517/14740338.2013.752813

11. Montandon SA, Jornayvaz FR. Effects of antidiabetic drugs on gut microbiota composition. Genes. (2017) 8:250. doi: 10.3390/genes8100250

12. Nguyen TL, Vieira-Silva S, Liston A, Raes J. How informative is the mouse for human gut microbiota research? Dis Model Mech. (2015) 8:116. doi: $10.1242 / \mathrm{dmm} .017400$

13. Pascale A, Marchesi N, Govoni S, Coppola A, Gazzaruso C. The role of gut microbiota in obesity, diabetes mellitus, and effect of metformin:

\section{SUPPLEMENTARY MATERIAL}

The Supplementary Material for this article can be found online at: https://www.frontiersin.org/articles/10.3389/fendo. 2020.573891/full\#supplementary-material

Supplementary Figure 1 | Risk of bias of randomized trials. (A) Each risk of bias item for each study. (B) Each risk of bias item across studies.

Supplementary Figure 2 | Risk of bias of quasi-experimental studies. (A) Each risk of bias item for each study. (B) Each risk of bias item across studies.

Supplementary Figure $\mathbf{3}$ | Risk of bias of observational studies. (A) Each risk of bias item for each study. (B) Each risk of bias item across studies.

Supplementary Figure 4 | Risk of bias of animal studies. (A) Each risk of bias item for each study. (B) Each risk of bias item across studies.

Supplementary Table 1 | Searching strategy.

Supplementary Table 2 | Characteristics of housing and dietary of studied animals.

Supplementary Table 3 | Effects of anti-hyperglycemia drugs on level of short-chain fatty acids (SCFAs).

Supplementary Table 4 | Effects of anti-hyperglycemia on bile acid levels.

new insights into old diseases. Curr Opin Pharmacol. (2019) 49:15. doi: 10.1016/j.coph.2019.03.011

14. Scells H, Zuccon G, CSIRO BK, Deacon A, Azzopardi L, Geva S. Integrating the framing of clinical questions via pico into the retrieval of medical literature for systematic reviews. In: Proceedings of the 2017 ACM on Conference on Information and Knowledge Management; 2017 November 6-10; Singapore. Association for Computing Machinery, New York, NY (2017). p. 2291-94.

15. Liberati A, Altman DG, Tetzlaff J, Mulrow C, Gotzsche PC, Ioannidis JP, et al. The PRISMA statement for reporting systematic reviews and meta-analyses of studies that evaluate healthcare interventions: explanation and elaboration. BMJ. (2009) 339:b2700. doi: 10.1136/bmj.b2700

16. Higgins JP, Altman DG, Gotzsche PC, Juni P, Moher D, Oxman AD, et al. The cochrane collaboration's tool for assessing risk of bias in randomised trials. BMJ. (2011) 343:d5928. doi: 10.1136/bmj.d5928

17. Sterne JA, Hernan MA, Reeves BC, Savovic J, Berkman ND, Viswanathan M, et al. ROBINS-I: a tool for assessing risk of bias in non-randomised studies of interventions. BMJ. (2016) 355:i4919. doi: 10.1136/bmj.i4919

18. Hooijmans CR, Rovers MM, de Vries RB, Leenaars M, Ritskes-Hoitinga M, Langendam MW. SYRCLE's risk of bias tool for animal studies. BMC Med Res Methodol. (2014) 14:43. doi: 10.1186/1471-2288-14-43

19. Ejtahed HS, Tito RY, Siadat SD, Hasani-Ranjbar S, Hoseini-Tavassol $\mathrm{Z}$, Rymenans L, et al. Metformin induces weight loss associated with gut microbiota alteration in non-diabetic obese women: a randomized double-blind clinical trial. Eur J Endocrinol. (2019) 180:165-76. doi: 10.1530/EJE-18-0826

20. Tong X, Xu J, Lian F, Yu X, Zhao Y, Xu L, et al. Structural alteration of gut microbiota during the amelioration of human type 2 diabetes with hyperlipidemia by metformin and a traditional Chinese herbal formula: a multicenter, randomized, open label clinical trial. mBio. (2018) 9:e0239217. doi: $10.1128 / \mathrm{mBio} .02392-17$

21. Wu H, Esteve E, Tremaroli V, Khan MT, Caesar R, Mannerås-Holm L, et al. Metformin alters the gut microbiome of individuals with treatment-naive type 2 diabetes, contributing to the therapeutic effects of the drug. Nat Med. (2017) 23:850-8. doi: $10.1038 / \mathrm{nm} .4345$

22. Bryrup T, Thomsen CW, Kern T, Allin KH, Brandslund I, Jørgensen NR, et al. Metformin-induced changes of the gut microbiota in healthy young men: results of a non-blinded, one-armed intervention study. Diabetologia. (2019) 62:1024-35. doi: 10.1007/s00125-019-4848-7

23. Huang F, Nilholm C, Roth B, Linninge C, Höglund P, Nyman M, et al. Anthropometric and metabolic improvements in human type 2 diabetes after introduction of an okinawan-based nordic diet are not associated with 
changes in microbial diversity or SCFA concentrations. Int J Food Sci Nutr. (2018) 69:729-40. doi: 10.1080/09637486.2017.1408059

24. Sun L, Xie C, Wang G, Wu Y, Wu Q, Wang X, et al. Gut microbiota and intestinal FXR mediate the clinical benefits of metformin. Nat Med. (2018) 24:1919-29. doi: 10.1038/s41591-018-0222-4

25. Elbere I, Kalnina I, Silamikelis I, Konrade I, Zaharenko L, Sekace $\mathrm{K}$, et al. Association of metformin administration with gut microbiome dysbiosis in healthy volunteers. PLOS ONE. (2018) 13:e204317. doi: 10.1371/journal.pone.0204317

26. Napolitano A, Miller S, Nicholls AW, Baker D, Van Horn S, Thomas E, et al. Novel gut-based pharmacology of metformin in patients with type 2 diabetes mellitus. PLoS ONE. (2014) 9:e100778. doi: 10.1371/journal.pone.0100778

27. Zhang F, Wang M, Yang J, Xu Q, Liang C, Chen B, et al. Response of gut microbiota in type 2 diabetes to hypoglycemic agents. Endocrine. (2019) 66:485-93. doi: 10.1007/s12020-019-02041-5

28. Barengolts E, Green SJ, Eisenberg Y, Akbar A, Reddivari B, Layden BT, et al. Gut microbiota varies by opioid use, circulating leptin and oxytocin in African American men with diabetes and high burden of chronic disease. PLoS ONE. (2018) 13:e0194171. doi: 10.1371/journal.pone.0194171

29. De La Cuesta-Zuluaga J, Mueller NT, Corrales-Agudelo V, Velásquez-Mejía EP, Carmona JA, Abad JM, et al. Metformin is associated with higher relative abundance of mucin-degrading akkermansia muciniphila and several shortchain fatty acid-producing microbiota in the gut. Diabetes Care. (2017) 40:54-62. doi: $10.2337 / \mathrm{dc} 16-1324$

30. Forslund K, Hildebrand F, Nielsen T, Falony G, Le Chatelier E, Sunagawa S, et al. Disentangling type 2 diabetes and metformin treatment signatures in the human gut microbiota. Nature. (2015) 528:262-6. doi: 10.1038/nature15766

31. Karlsson FH, Tremaroli V, Nookaew I, Bergström G, Behre CJ, Fagerberg B, et al. Gut metagenome in European women with normal, impaired and diabetic glucose control. Nature. (2013) 498:99-103. doi: 10.1038/nature12198

32. Gu Y, Wang X, Li J, Zhang Y, Zhong H, Liu R, et al. Analyses of gut microbiota and plasma bile acids enable stratification of patients for antidiabetic treatment. Nat Commun. (2017) 8:1785. doi: 10.1038/s41467-017-01682-2

33. Su B, Liu H, Li J, Sunli Y, Liu B, Liu D, et al. Acarbose treatment affects the serum levels of inflammatory cytokines and the gut content of bifidobacteria in Chinese patients with type 2 diabetes mellitus. J Diabetes. (2015) 7:72939. doi: $10.1111 / 1753-0407.12232$

34. Wang Z, Saha S, Van Horn S, Thomas E, Traini C, Sathe G, et al. Gut microbiome differences between metformin- and liraglutide-treated T2DM subjects. Endocrinol Diabetes Metab. (2018) 1:e00009. doi: 10.1002/edm2.9

35. Zhang $\mathrm{W}, \mathrm{Xu} \mathrm{JH}, \mathrm{Yu} \mathrm{T}$, Chen QK. Effects of berberine and metformin on intestinal inflammation and gut microbiome composition in $\mathrm{db} / \mathrm{db}$ mice. Biomed Pharmacother. (2019) 118:109131. doi: 10.1016/j.biopha.2019.109131

36. Xue J, Li X, Liu $\mathrm{P}$, Li K, Sha $\mathrm{L}$, Yang $\mathrm{X}$, et al. Inulin and metformin ameliorate polycystic ovary syndrome via antiinflammation and modulating gut microbiota in mice. Endocr J. (2019) 66:859-70. doi: 10.1507/endocrj.EJ18-0567

37. Ryan PM, Patterson E, Carafa I, Mandal R, Wishart DS, Dinan TG, et al. Metformin and dipeptidase peptidyl-4 inhibitor differentially modulate the intestinal microbiota and plasma metabolome of metabolically dysfunctional mice. Can J Diabetes. (2019) 44:146-55.e2. doi: 10.1016/j.jcjd.2019.05.008

38. Ji S, Wang L, Li L. Effect of metformin on short-term high-fat dietinduced weight gain and anxiety-like behavior and the gut microbiota. Front Endocrinol. (2019) 10:704. doi: 10.3389/fendo.2019.00704

39. Dong TS, Chang HH, Hauer M, Lagishetty V, Katzka W, Rozengurt E, et al. Metformin alters the duodenal microbiome and decreases the incidence of pancreatic ductal adenocarcinoma promoted by dietinduced obesity. Am J Physiol Gastrointest Liver Physiol. (2019) 317:G76372. doi: 10.1152/ajpgi.00170.2019

40. Brandt A, Hernández-Arriaga A, Kehm R, Sánchez V, Jin CJ, Nier A, et al. Metformin attenuates the onset of non-alcoholic fatty liver disease and affects intestinal microbiota and barrier in small intestine. Sci Rep. (2019) 9:6668. doi: 10.1038/s41598-019-43228-0

41. Adeshirlarijaney A, Zou J, Tran H, Chassaing B, Gewirtz AT. Amelioration of metabolic syndrome by metformin associates with reduced indices of low-grade inflammation independently of the gut microbiota. Am J Physiol Endocrinol Metab. (2019) 317:E1121-30. doi: 10.1152/ajpendo.00245.2019
42. Xu M, Yue RS, Yang MY, Yang X, Wu TC, Li JN. Effects of banxia xiexin decoction on intestinal flora and inflammatory factors of diabetic gastroparesis rats. Chin Trad Herbal Drugs. (2018) 49:3056-61. doi: 10.7501/j.issn.0253-2670.2018.13.015

43. Bauer PV, Duca FA, Waise TMZ, Rasmussen BA, Abraham MA, Dranse HJ, et al. Metformin alters upper small intestinal microbiota that impact a glucose-SGLT1-sensing glucoregulatory pathway. Cell Metab. (2018) 27:10117.e5. doi: 10.1016/j.cmet.2017.09.019

44. Zheng J, Li $\mathrm{H}$, Zhang $\mathrm{X}$, Jiang $\mathrm{M}$, Luo $\mathrm{C}, \mathrm{Lu} \mathrm{Z}$, et al. Prebiotic mannan-oligosaccharides augment the hypoglycemic effects of metformin in correlation with modulating gut microbiota. J Agric Food Chem. (2018) 66:5821-31. doi: 10.1021/acs.jafc.8b00829

45. Wang JH, Bose S, Shin NR, Chin YW, Choi YH, Kim H. Pharmaceutical impact of houttuynia cordata and metformin combination on high-fat-dietinduced metabolic disorders: link to intestinal microbiota and metabolic endotoxemia. Front Endocrinol. (2018) 9:620. doi: 10.3389/fendo.2018.00620

46. Ma W, Chen J, Meng Y, Yang J, Cui Q, Zhou Y. Metformin alters gut microbiota of healthy mice: implication for its potential role in gut microbiota homeostasis. Front Microbiol. (2018) 9:1336. doi: 10.3389/fmicb.2018.01336

47. Liu G, Bei J, Liang L, Yu G, Li L, Li Q. Stachyose improves inflammation through modulating gut microbiota of high-fat diet/streptozotocininduced type 2 diabetes in rats. Mol Nutr Food Res. (2018) 62:e1700954. doi: 10.1002/mnfr.201700954

48. Lee H, Lee Y, Kim J, An J, Lee S, Kong H, et al. Modulation of the gut microbiota by metformin improves metabolic profiles in aged obese mice. Gut Microbes. (2018) 9:155-65. doi: 10.1080/19490976.2017.1405209

49. Shin NR, Bose S, Wang JH, Ansari A, Lim SK, Chin YW, et al. Flos lonicera combined with metformin ameliorates hepatosteatosis and glucose intolerance in association with gut microbiota modulation. Front Microbiol. (2017) 8:2271. doi: 10.3389/fmicb.2017.02271

50. Wang JH, Bose S, Lim SK, Ansari A, Chin YW, Choi HS, et al. Houttuynia cordata facilitates metformin on ameliorating insulin resistance associated with gut microbiota alteration in OLETF rats. Genes. (2017) 8:239. doi: $10.3390 /$ genes 8100239

51. Han K, Bose S, Wang JH, Lim SK, Chin YW, Kim YM, et al. In vivo therapeutic effect of combination treatment with metformin and scutellaria baicalensis on maintaining bile acid homeostasis. PLOS ONE. (2017) 12:e0182467. doi: 10.1371/journal.pone.0182467

52. Salomäki-Myftari H, Vähätalo LH, Ailanen L, Pietilä S, Laiho A, Hänninen A, et al. Neuropeptide $Y$ overexpressing female and male mice show divergent metabolic but not gut microbial responses to prenatal metformin exposure. PLoS ONE. (2016) 11:e0163805. doi: 10.1371/journal.pone.0163805

53. Zhou ZY, Ren LW, Zhan P, Yang HY, Chai DD, Yu ZW. Metformin exerts glucose-lowering action in high-fat fed mice via attenuating endotoxemia and enhancing insulin signaling. Acta Pharmacol Sin. (2016) 37:106375. doi: 10.1038/aps.2016.21

54. Zhang X, Zhao Y, Xu J, Xue Z, Zhang M, Pang X, et al. Modulation of gut microbiota by berberine and metformin during the treatment of high-fat diet-induced obesity in rats. Sci Rep. (2015) 5:14405. doi: 10.1038/srep14405

55. Lee H, Ko G. Effect of metformin on metabolic improvement and gut microbiota. Appl Environ Microbiol. (2014) 80:593543. doi: 10.1128/AEM.01357-14

56. Shin NR, Lee JC, Lee HY, Kim MS, Whon TW, Lee MS, et al. An increase in the Akkermansia spp. population induced by metformin treatment improves glucose homeostasis in diet-induced obese mice. Gut. (2014) 63:727-35. doi: 10.1136/gutjnl-2012-303839

57. Pyra KA, Saha DC, Reimer RA. Prebiotic fiber increases hepatic acetyl CoA carboxylase phosphorylation and suppresses glucosedependent insulinotropic polypeptide secretion more effectively when used with metformin in obese rats. J Nutr. (2012) 142:213-20. doi: 10.3945/jn.111.147132

58. Zhao J, Li Y, Sun M, Xin L, Wang T, Wei L, et al. The Chinese herbal formula shenzhu tiaopi granule results in metabolic improvement in type 2 diabetic rats by modulating the gut microbiota. Evid Based Complement Altern Med. (2019) 2019:6976394. doi: 10.1155/2019/6976394

59. Smith BJ, Miller RA, Ericsson AC, Harrison DC, Strong R, Schmidt TM. Changes in the gut microbiome and fermentation products concurrent 
with enhanced longevity in acarbose-treated mice. BMC Microbiol. (2019) 19:130. doi: 10.1186/s12866-019-1494-7

60. Liao X, Song L, Zeng B, Liu B, Qiu Y, Qu H, et al. Alteration of gut microbiota induced by DPP-4i treatment improves glucose homeostasis. EBioMedicine. (2019) 44:665-74. doi: 10.1016/j.ebiom.2019.03.057

61. Baxter NT, Lesniak NA, Sinani H, Schloss PD, Koropatkin NM. The glucoamylase inhibitor acarbose has a diet-dependent and reversible effect on the murine gut microbiome. mSphere. (2019) 4:e00528-18. doi: 10.1128/mSphere.00528-18

62. Xu GD, Cai L, Ni YS, Tian SY, Lu YQ, Wang LN, et al. Comparisons of effects on intestinal short-chain fatty acid concentration after exposure of two glycosidase inhibitors in mice. Biol Pharm Bull. (2018) 41:102433. doi: 10.1248/bpb.b17-00978

63. Do HJ, Lee YS, Ha MJ, Cho Y, Yi H, Hwang YJ, et al. Beneficial effects of voglibose administration on body weight and lipid metabolism via gastrointestinal bile acid modification. Endocr J. (2016) 63:691702. doi: $10.1507 /$ endocrj.EJ15-0747

64. Kishida Y, Okubo H, Ohno H, Oki K, Yoneda M. Effect of miglitol on the suppression of nonalcoholic steatohepatitis development and improvement of the gut environment in a rodent model. J Gastroenterol. (2017) 52:118091. doi: 10.1007/s00535-017-1331-4

65. Madsen MSA, Holm JB, Pallejà A, Wismann P, Fabricius K, Rigbolt K, et al. Metabolic and gut microbiome changes following GLP-1 or dual GLP-1/GLP2 receptor agonist treatment in diet-induced obese mice. Sci Rep. (2019) 9:15582. doi: 10.1038/s41598-019-52103-x

66. Zhao L, Chen Y, Xia F, Abudukerimu B, Zhang W, Guo Y, et al. A glucagon-like peptide-1 receptor agonist lowers weight by modulating the structure of gut microbiota. Front Endocrinol. (2018) 9:233. doi: 10.3389/fendo.2018.00233

67. Yuan X, Ni H, Chen X, Feng X, Wu Q, Chen J. Identification of therapeutic effect of glucagon-like peptide 1 in the treatment of STZ-induced diabetes mellitus in rats by restoring the balance of intestinal flora. J Cell Biochem. (2018) 119:10067-74. doi: 10.1002/jcb.27343

68. Moreira GV, Azevedo FF, Ribeiro LM, Santos A, Guadagnini D, Gama P, et al. Liraglutide modulates gut microbiota and reduces NAFLD in obese mice. $J$ Nutr Biochem. (2018) 62:143-54. doi: 10.1016/j.jnutbio.2018.07.009

69. Zhang Q, Xiao X, Zheng J, Li M, Yu M, Ping F, et al. Featured article: structure moderation of gut microbiota in liraglutide-treated diabetic male rats. Exp Biol Med. (2018) 243:34-44. doi: 10.1177/1535370217743765

70. Wang L, Li P, Tang Z, Yan X, Feng B. Structural modulation of the gut microbiota and the relationship with body weight: compared evaluation of liraglutide and saxagliptin treatment. Sci Rep. (2016) 6:33251. doi: $10.1038 /$ srep33251

71. Kaya D, Kaji K, Tsuji Y, Yamashita S, Kitagawa K, Ozutsumi T, et al. Tgr5 activation modulates an inhibitory effect on liver fibrosis development mediated by anagliptin in diabetic rats. Cells. (2019) 8:1153. doi: $10.3390 /$ cells 8101153

72. Dennison CA, Eslinger AJ, Reimer RA. Preconception prebiotic and sitagliptin treatment in obese rats affects pregnancy outcomes and offspring microbiota, adiposity, and glycemia. Front Endocrinol. (2017) 8:301. doi: $10.3389 /$ fendo. 2017.00301

73. Yan X, Feng B, Li P, Tang Z, Wang L. Microflora disturbance during progression of glucose intolerance and effect of sitagliptin: an animal study. J Diabetes Res. (2016) 2016:2093171. doi: 10.1155/2016/2093171

74. Olivares M, Neyrinck AM, Pötgens SA, Beaumont M, Salazar N, Cani PD, et al. The DPP-4 inhibitor vildagliptin impacts the gut microbiota and prevents disruption of intestinal homeostasis induced by a Western diet in mice. Diabetologia. (2018) 61:1838-48. doi: 10.1007/s00125-018-4647-6

75. Li L, Xu S, Guo T, Gong S, Zhang C. Effect of dapagliflozin on intestinal flora in mafa-deficient mice. Curr Pharm Des. (2018) 24:322331. doi: 10.2174/1381612824666180912143434

76. Lee DM, Battson ML, Jarrell DK, Hou S, Ecton KE, Weir TL, et al. SGLT2 inhibition via dapagliflozin improves generalized vascular dysfunction and alters the gut microbiota in type 2 diabetic mice. Cardiovasc Diabetol. (2018) 17:62. doi: 10.1186/s12933-018-0708-x
77. Mishima E, Fukuda S, Kanemitsu Y, Saigusa D, Mukawa C, Asaji K, et al. Canagliflozin reduces plasma uremic toxins and alters the intestinal microbiota composition in a chronic kidney disease mouse model. Am J Physiol Renal Physiol. (2018) 315:F824-33. doi: 10.1152/ajprenal.003 14.2017

78. Li Y, Han L, Xu M, Guo J, Feng M, Wang X. The primary research on the gut microbes in KKAy mice. Indian J Microbiol. (2014) 54:129. doi: 10.1007/s12088-013-0410-3

79. Wang LY, Wang S, Wang Y, Ruan KF, Feng Y. Effect of MDG-1 on oral glucose tolerance and intestinal microecological balance in diabetic mice. World Chin J Digestol. (2011) 19:2058-62. doi: 10.11569/wcjd.v19.i19.2058

80. Bytzer P, Talley NJ, Jones MP, Horowitz M. Oral hypoglycaemic drugs and gastrointestinal symptoms in diabetes mellitus. Aliment Pharmacol Ther. (2001) 15:137-42. doi: 10.1046/j.1365-2036.2001.00896.x

81. Sedighi M, Razavi S, Navab-Moghadam F, Khamseh ME, Alaei-Shahmiri F, Mehrtash A, et al. Comparison of gut microbiota in adult patients with type 2 diabetes and healthy individuals. Microb Pathog. (2017) 111:3629. doi: 10.1016/j.micpath.2017.08.038

82. Candela M, Biagi E, Soverini M, Consolandi C, Quercia S, Severgnini $\mathrm{M}$, et al. Modulation of gut microbiota dysbioses in type 2 diabetic patients by macrobiotic Ma-Pi 2 diet. Br J Nutr. (2016) 116:8093. doi: 10.1017/S0007114516001045

83. Derrien M, Belzer C, de Vos WM. Akkermansia muciniphila and its role in regulating host functions. Microb Pathog. (2017) 106:17181. doi: $10.1016 /$ j.micpath.2016.02.005

84. Wang J, Li W, Wang C, Wang L, He T, Hu H, et al. Enterotype bacteroides is associated with a high risk in patients with diabetes: a pilot study. J Diabetes Res. (2020) 2020:6047145. doi: 10.1155/2020/6047145

85. Cani PD, Possemiers S, Van de Wiele T, Guiot Y, Everard A, Rottier O, et al. Changes in gut microbiota control inflammation in obese mice through a mechanism involving GLP-2-driven improvement of gut permeability. Gut. (2009) 58:1091-103. doi: 10.1136/gut.2008.165886

86. Ley RE, Backhed F, Turnbaugh P, Lozupone CA, Knight RD, Gordon JI. Obesity alters gut microbial ecology. Proc Natl Acad Sci USA. (2005) 102:11070-5. doi: 10.1073/pnas.0504978102

87. Pedersen C, Ijaz UZ, Gallagher E, Horton F, Ellis RJ, Jaiyeola E, et al. Fecal enterobacteriales enrichment is associated with increased in vivo intestinal permeability in humans. Physiol Rep. (2018) 6:e13649. doi: 10.14814/phy2.13649

88. Rizzatti G, Lopetuso LR, Gibiino G, Binda C, Gasbarrini A. Proteobacteria: a common factor in human diseases. Biomed Res Int. (2017) 2017:9351507. doi: 10.1155/2017/9351507

89. King $\mathrm{CH}$, Desai $\mathrm{H}$, Sylvetsky AC, LoTempio J, Ayanyan S, Carrie J, et al. Baseline human gut microbiota profile in healthy people and standard reporting template. PLoS ONE. (2019) 14:e0206484. doi: 10.1371/journal.pone.0206484

90. Dalile B, Van Oudenhove L, Vervliet B, Verbeke K. The role of short-chain fatty acids in microbiota-gut-brain communication. Nat Rev Gastroenterol Hepatol. (2019) 16:461-78. doi: 10.1038/s41575-019-0157-3

91. Ridlon JM, Kang DJ, Hylemon PB, Bajaj JS. Bile acids and the gut microbiome. Curr Opin Gastroenterol. (2014) 30:3328. doi: 10.1097/MOG.0000000000000057

Conflict of Interest: The authors declare that the research was conducted in the absence of any commercial or financial relationships that could be construed as a potential conflict of interest.

Copyright (c) $2020 \mathrm{Cao}, \mathrm{Wu}, \mathrm{Hsu}$, Chang, Chou, Lin, Liao, Lin, Yang and Lin. This is an open-access article distributed under the terms of the Creative Commons Attribution License (CC BY). The use, distribution or reproduction in other forums is permitted, provided the original author(s) and the copyright owner(s) are credited and that the original publication in this journal is cited, in accordance with accepted academic practice. No use, distribution or reproduction is permitted which does not comply with these terms. 